\title{
EXISTENCE AND LARGE TIME BEHAVIOUR FOR A STOCHASTIC MODEL OF MODIFIED MAGNETOHYDRODYNAMIC EQUATIONS
}

\author{
PAUL ANDRÉ RAZAFIMANDIMBY AND MAMADOU SANGO
}

\begin{abstract}
In this paper we study a system of nonlinear Stochastic Partial Differential equations describing the motion of turbulent Non-Newtonian media in the presence of fluctuating magnetic field. The system is basically obtained by a coupling of the dynamical equations of a NonNewtonian fluids having $p$-structure and the Maxwell equations. We mainly show the existence of weak martingale solutions and their exponential decay when time goes to infinity.
\end{abstract}

\section{INTRODUCTION}

Stochastic Partial Differential Equations (SPDEs for short) have now become very important tools in Hydrodynamics. They are used in the mathematical investigation towards the understanding of turbulent motions of fluids. The SPDEs governing turbulent fluids are obtained by adding noise terms to deterministic models. This approach is basically motivated by Reynolds' work which stipulates that the velocity of a fluid particle in turbulent regime is composed of slow (deterministic) and fast (stochastic) components. While this belief was based on empirical and experimental data, Rozovskii and Mikulevicius were able to derive the models rigorously in their recent work [43], thereby confirming the importance of this approach in hydrodynamic turbulence. Indeed, it is pointed out in [43] (see also [32]) that some rigorous information on questions in Turbulence might be obtained from stochastic versions of the equations of fluid dynamics.

The mathematical study of SPDEs for hydrodynamics was initiated in the early 1970's in the papers of Bensoussan and Temam [4], since then stochastic Navier-Stokes equations and SPDEs in general have been the object of intensive research which has generated several important results. We refer to, among others, [1], [8], [10], [11], [17], [19], [20], [43], [44],[50],[51], [52], [53],[59],[61].

Magnetohydrodynamics (MHD) is a branch of continuum mechanics which studies the motion of conducting fluids in the presence of magnetic fields. The system of Partial Differential Equations (PDEs) in MHD are basically obtained through the coupling of the dynamical equations of the fluids with the Maxwell's equations which are used to take into account the effect of the Lorentz force due to the magnetic field (see for example [15]). Magnetohydrodynamics plays essential role in Astrophysics, Geophysics, Plasma Physics, the magnetic confinement device Tokamak in Thermonuclear Physics, and in many other branches of applied sciences. In these areas turbulent magnetohydrodynamic flows which are usually due to magnetic-field fluctuations are typical. Deterministic models of MHD have been the focus of investigation by many mathematicians. Several important results have been obtained, for instance in [34], [69], [62], [22], [18], [40], [41]; just to cite a few relevant papers. The reader can consult [23] for a recent and detailed account in the mathematical investigation of hydrodynamic turbulence. Many scientists have also considered stochastic models for MHD by adding noise terms to the dynamical equations of the fluids and Maxwell equations representing the magnetic-field fluctuations. The stochastic MHD equations were investigated in [67, 2, 60]. The authors in [67], [2] consider additive noises.

Date: April 6, 2015. 
Using Galerkin's approximation and compactness method, the author in [60] proved the existence of martingale weak solutions for the stochastic MHD equations in the presence of nonlinear multiplicative noise which do not satisfy the Lipschitz condition.

Due to the conventional belief that the Navier-Stokes equations are an accurate model for the motion of incompressible fluids in many practical situations, the majority of the above work have assumed that the fluids are Newtonian. However, there are a lot of conducting materials appearing in many practical and theoretical situations that cannot be characterized by Newtonian fluids. To describe these media one generally has to use (conducting) fluids models that allow the stress to be a nonlinear function of the strain rate. Fluids in the latter class are called Non-Newtonian fluids. We refer for example to the introduction of Biskamp's book [6] for some examples of these Non-Newtonian conducting fluids. These facts motivated us to consider in the present manuscript a class of stochastic modified MHD equations which allows the constitutive law of the conducting fluids to exhibit a nonlinear relationship between the stress tensor and the strain rate. More precisely, for a final time $T>0$ and a sufficiently smooth bounded domain $\mathcal{Q}$ in $\mathbb{R}^{n}(n=2,3)$ we describe the motion of randomly forced Non-Newtonian conducting fluids in a fluctuating magnetic field by the following system of stochastic partial differential equations:

$$
\left\{\begin{array}{l}
d \mathbf{u}+(-\operatorname{div} \mathbf{T}+\mathbf{u} \cdot \nabla \mathbf{u}+\mu \mathbf{B} \times \operatorname{curl} \mathbf{B}+\nabla P) d t=f_{1}(\mathbf{u}, \mathbf{B}, t) d t+g_{1}(\mathbf{u}, \mathbf{B}, t) d W_{1}, \\
d \mathbf{B}+(S \operatorname{curl} \operatorname{curl} \mathbf{B}+\mu \mathbf{u} \cdot \nabla \mathbf{B}-\mu \mathbf{B} \cdot \nabla \mathbf{u}) d t=f_{2}(\mathbf{u}, \mathbf{B}, t) d t+g_{2}(\mathbf{u}, \mathbf{B}, t) d W_{2}, \\
\operatorname{div} \mathbf{u}=\operatorname{div} \mathbf{B}=0, \\
\mathbf{u}=0 \text { on } \partial \mathcal{Q} \times(0, T), \\
\mathbf{B} \cdot n=\operatorname{curl} \mathbf{B} \times n=0 \text { on } \partial \mathcal{Q} \times(0, T), \\
\mathbf{u}(0)=\mathbf{u}_{0}, \mathbf{B}(0)=\mathbf{B}_{0},
\end{array}\right.
$$

where $\mathbf{u}=\left(u_{i} ; i=1, \ldots, n\right), \mathbf{B}=\left(B_{i} ; i=1, \ldots, n\right)$ and $P$ are unknown random fields defined on $\mathcal{Q} \times[0, T]$, representing, respectively, the fluid velocity, the magnetic field and the pressure, at each point of $\mathcal{Q} \times[0, T]$. $S$ and $\mu$ are positive constants depending on the Reynolds numbers of the fluid and magnetic fields, and the Hartman number. The terms $f_{i}(\mathbf{u}, \mathbf{B}, t)$ and $g_{i}(\mathbf{u}, \mathbf{B}, t) d W_{i}$ $(\mathrm{i}=1,2)$ are external forces depending on $u$ and $B$, where $W_{i}$ are cylindrical Wiener processes evolving on two Hilbert spaces $\mathbb{K}_{i}$. We assume they are mutually independent and identically distributed. The quantities $\mathbf{u}_{0}$ and $\mathbf{B}_{0}$ are given non random initial velocity and magnetic field, respectively. Finally, $\mathbf{T}$ designates the extra stress tensor of the Non-Newtonian fluid and we suppose that there exists a potential $\Sigma: \mathbb{R}_{s y m}^{n \times n} \rightarrow \mathbb{R}_{0}^{+}$and constants $\nu_{1}, \nu_{2}$ such that for some $p>1$ and for all $l, k, i, j=1,2, \ldots, n, \mathbf{D}, \mathbf{E} \in \mathbb{R}_{s y m}^{n \times n}$ :

$$
\begin{aligned}
\Sigma(0) & =0, \quad \frac{\partial \Sigma(0)}{\partial D_{k l}}=\partial_{k l} \Sigma(0)=0, \quad T_{k l}(\mathbf{D})=\partial_{k l} \Sigma(\mathbf{D}), \\
\partial_{i j} \partial_{k l} \Sigma(\mathbf{D}) E_{i j} E_{k l} & \geq \nu_{1}(1+\mathbf{D})^{p-2}|\mathbf{E}|^{2}, \\
\partial_{i j} \partial_{k l} \Sigma(\mathbf{D}) & \leq \nu_{2}\left(1+|\mathbf{D}|^{2}\right)^{p-2} .
\end{aligned}
$$

Here

$$
\mathbb{R}_{\text {sym }}^{n \times n}=\left\{\mathbf{D} \in \mathbb{R}^{n \times n}: D_{i j}=D_{j i}, i, j=1,2, \ldots, n\right\} .
$$

The structure of the nonlinearity of problem (1) introduces a number of interesting features which are not present in their Newtonian counterparts such as the basic MHD or the NavierStokes equations; we refer for instance to the papers [36], [38], [39] which deal with interesting mathematical questions arising in similar fluids in the deterministic case. Besides the usual nonlinear terms of the MHD equations, (1) contains another nonlinear term of $p$-structure which exhibits the non-linear relationships between the reduced stress and the rate of strain of the conducting fluids. Because of this, the analysis of the behavior of the MHD model (1) tends to be much more complicated and subtle than that of the Newtonian MHD equations. 
In the deterministic case, that is when $g_{1}, g_{2} \equiv 0$, a variant of (1) (the tensor $\mathbf{T}$ is replaced by the $p$-Laplacian) and its stationary version were initially investigated by Samokhin in [58], [57], [55], and [56]. Later on Gunzburger and his coworkers in [24] and [25] considered a more general model by taking a tensor $\mathbf{T}$ which satisfies the assumption we have made above. The paper [25] dealt with the control of (1) and [58], [57], [55], [56], and [24] addressed the existence and uniqueness results of weak solution of (1). To the best of our knowledge, there is no known results for the stochastic equations (1). The purpose of the present paper is to prove some results related to problem (1) which are the stochastic analog of some of those obtained in $[58,24]$ for the deterministic case. The following two points are our main goals:

(1) We prove the existence of martingale weak solution for the stochastic system (1). We consider a sufficiently general forcing consisting of a regular part and a stochastic part both depending nonlinearly on the velocity of the fluids and the magnetic field, and we do not require the functions involved in the forcing term to satisfy the Lipschitz condition. The method for the proof uses a blending of Galerkin, compactness, and monotonicity methods. We closely follow the article [24] and the book [42] but we do not use the usual martingale representation argument.

(2) After obtaining the existence of a martingale weak solution of our model, we turn our attention to the study of its asymptotic behavior as the time $t$ is large. For this purpose, we study the decay of the martingale weak solutions as time goes to infinity. We mainly prove that under some conditions on the forcing terms $f_{i}$ and $g_{i}, i=1,2$ the couple $(\mathbf{u} ; \mathbf{B})$ converges to zero almost surely exponentially. To prove this result we mainly follow the idea in [13] and [14]

The stochastic Navier-Stokes and MHD equations for Newtonian fluids have been extensively studied. In addition to the papers we cited earlier we would like also to mention the recent article [45] in which the spatial domain is allowed to be unbounded, and the coefficients of the noise depend multiplicatively on both the velocity field and its spatial derivatives. The key ingredients of the proof of existence of martingale solutions in [45] (see also [11], [44]) are Galerkin method, the use of Fréchet space, tightness criteria in nonmetrizable spaces and a version of the Skorokhod Theorem in non-metric spaces. The framework of [45] is very general as it allows to treat the stochastic Navier-Stokes, magneto-hydrodynamic (MHD) and the Boussinesq equations driven by the sum of Wiener and Compensated Poisson random measure. However, the problem we treat here do not fall into the general framework of [45] or previous work about stochastic NavierStokes and MHD equations. The main reason is the presence of the additional nonlinear term of $p$-structure which makes the mathematical analysis of (1) difficult and subtle. Our method relies on Galerkin approximation and compactness described in the books $[42,47]$ and some other papers such as [9] and [24]. We also rely heavily on Korn's inequality which, to our knowledge, only holds in bounded domain.

As far as we know the present article is the first to deal with (1). In this sense, many topics and problems related to (1) still stand opened. Some examples of challenges we may address in future research are the existence of weak solution for $p \in(1,2)$, the uniqueness of such weak solutions. We may also want to study the existence and uniqueness of the invariant measure (whenever it is possible) which, we believe, does not follow in an obvious and straightforward way from the most recent results about ergodicity of SPDEs (see, for instance, [26] and [28]). In fact, we do not know much about the long time behaviour of (1) even in the deterministic case. These few examples of research topics are taken as an analogy of the problems still unsolved in the mathematical theory of Non-Newtonian as reported in [3], [36] and [37]. The investigations of these problems may lead to new and important results that will be useful for further development in the theory of deterministic and stochastic PDEs. But due to the nature of the nonlinear terms involved in (1) all of these questions are very difficult and beyond the scope of this paper, thus 
we will just limit ourselves with giving a suitable mathematical setting for (1) and partial results related to the dynamics of the weak solutions. However, we hope that our work will find its applications elsewhere or at least motivate further research in the study of stochastic model for Non-Newtonian MHD.

The paper is structured as follows. In Section 2, we gather all the necessary tools and the hypotheses. In section 3 we state the result for the existence of weak probabilistic solution and we prove it by means of Galerkin methods and probabilistic and analytic compactness results. The exponential asymptotic behavior of these weak solutions are studied in the last section.

\section{Preliminary: Notations and hypotheses}

In this section we introduce the necessary notations and most of the hypotheses relevant for our analysis.

2.1. The deterministic framework. We introduce some notations and background following the mathematical theory of hydrodynamic equations such as Navier-Stokes equations (NSE) or MHD equations. For any $q \in[1, \infty)$, we denote by $\mathbb{L}^{q}(\mathcal{Q})$ and $\mathbb{W}^{m, q}(\mathcal{Q})$ the space of functions taking values in $\mathbb{R}^{n}$ such that each component belongs to the Lebesgue spaces $L^{q}(\mathcal{Q})$ and the Sobolev spaces $W^{m, p}(\mathcal{Q})$, respectively. For $q=2$ we denote $\mathbb{W}^{m, q}(\mathcal{Q})$ by $\mathbb{H}^{m}(\mathcal{Q})$. We denote by $|\cdot|$ the $\mathbb{L}^{2}$-norm, and by $(.,$.$) the \mathbb{L}^{2}$-inner product. The norm of $\mathbb{W}^{q, m}(\mathcal{Q})$ is denoted by $\|\cdot\|_{m, q}$. We denote by $\mathbb{C}_{c}^{\infty}(\mathcal{Q})$ the space of functions $\mathbf{u} \in \mathbb{C}^{\infty}(\mathcal{Q})$ with compact supporrt. Let $p \in(1, \infty)$, following [62] we introduce the following spaces

$$
\begin{aligned}
\mathcal{V}_{1} & =\left\{\mathbf{u} \in \mathbb{C}_{c}^{\infty}(\mathcal{Q}): \operatorname{div} \mathbf{u}=0\right\}, \\
\mathbb{H}_{1} & =\left\{\mathbf{u} \in \mathbb{L}^{2}(\mathcal{Q}): \operatorname{div} \mathbf{u}=0, \mathbf{u} \cdot n=0 \text { on } \partial \mathcal{Q}\right\}, \\
\mathbb{V}_{1, p} & =\left\{\mathbf{u} \in \mathbb{W}^{1, p}: \operatorname{div} \mathbf{u}=0, \mathbf{u}=0 \text { on } \partial \mathcal{Q}\right\}, \\
\mathbb{V}_{1} & =\left\{\mathbf{u} \in \mathbb{H}^{1}(\mathcal{Q}): \operatorname{div} \mathbf{u}=0, \mathbf{u}=0 \text { on } \partial \mathcal{Q}\right\} .
\end{aligned}
$$

We also set

$$
\begin{aligned}
\mathcal{V}_{2} & =\left\{\mathbf{B} \in \mathbb{C}^{\infty}(\overline{\mathcal{Q}}): \operatorname{div} \mathbf{B}=0 ; \mathbf{B} \cdot n=0 \text { on } \partial \mathcal{Q}\right\} \\
\mathbb{H}_{2} & =\text { the closure of } \mathcal{V}_{2} \text { in } \mathbb{L}^{2}(\mathcal{Q}) \\
\mathbb{V}_{2} & =\left\{\mathbf{B} \in \mathbb{H}^{1}(\mathcal{Q}): \operatorname{div} \mathbf{B}=0 ; \mathbf{B} \cdot n=0 \text { on } \partial \mathcal{Q}\right\}
\end{aligned}
$$

Note that

$$
\mathbb{H}_{1}=\mathbb{H}_{2} \text {. }
$$

The spaces $\mathbb{H}_{i}, i=1,2$ are equipped with the scalar product and norm induced by $\mathbb{L}^{2}(\mathcal{Q})$.

Thanks to Poincaré's inequality we can endow the space $\mathbb{V}_{1, p}$ with the norm $\|\mathbf{u}\|_{1, p}$ defined by

$$
\|\mathbf{u}\|_{1, p}^{p}=\int_{\mathcal{Q}}|\nabla \mathbf{u}|^{p} d x .
$$

This norm is equivalent to the usual $\mathbb{W}^{1, p}$-norm on $\mathbb{V}_{1, p}$.

We equip the space $\mathbb{V}_{1}$ with the norm $\|\cdot\|_{1}$ generated by the scalar product

$$
((\mathbf{u}, \mathbf{v}))_{1}=\int_{\mathcal{Q}} \nabla \mathbf{u} \cdot \nabla \mathbf{v} d x
$$

Owing to Poincaré's inequality, $\|\cdot\|_{1}$ and the usual $\mathbb{H}^{1}(\mathcal{Q})$-norm are equivalent on $\mathbb{V}_{1}$.

On $\mathbb{V}_{2}$ we define the scalar product

$$
((\mathbf{u}, \mathbf{v}))_{2}=(\operatorname{curl} \mathbf{u}, \operatorname{curl} \mathbf{v}),
$$

which is equivalent to the usual scalar product of $\mathbb{H}^{1}(\mathcal{Q})$. 
Let

$$
\begin{gathered}
\mathbb{V}=\mathbb{V}_{1, p} \times \mathbb{V}_{2}, \\
\mathbb{H}=\mathbb{H}_{1} \times \mathbb{H}_{2} .
\end{gathered}
$$

The space $\mathbb{H}$ has the structure of a Hilbert space when equipped with the scalar product

$$
(\Phi, \Psi)=(\mathbf{u}, \mathbf{v})+(\mathbf{B}, \mathbf{C})
$$

for $\Phi=(\mathbf{u} ; \mathbf{B}), \Psi=(\mathbf{v} ; \mathbf{C}) \in \mathbb{H}$.

The space $\mathbb{V}$ is a Banach space with norm

for $\Phi=(\mathbf{u} ; \mathbf{B}) \in \mathbb{V}$.

$$
\|\Phi\|_{\mathbb{V}}=\|\mathbf{u}\|_{1, p}+\|\mathbf{B}\|_{2}
$$

Remark 2.1. Note that this norm is equivalent to any norm of the form

$$
[[\Phi]]_{\mathbb{V}}=C_{1}\|\mathbf{u}\|_{1, p}+C_{2}\|\mathbf{B}\|_{2}
$$

where the constants $C_{1}, C_{2}$ depend only on $S, \operatorname{mes}(\mathcal{Q}), p$. Here mes $(\mathcal{Q})$ denotes the Lebesgue measure of $\mathcal{Q}$.

Throughout this work we set

$$
\|\Phi\|_{\mathbb{V}}^{p, 2}=\|\mathbf{u}\|_{1, p}^{p}+\|\mathbf{B}\|_{2}^{2} .
$$

For any Banach space $X$ we denote by $X^{*}$ its dual space and $\langle\phi, \mathbf{u}\rangle$ the value of $\phi \in X^{*}$ on $\mathbf{u} \in X$.

Let $\mathcal{A}_{2}$ be the bounded linear operator from $\mathbb{V}_{2}$ taking values into $\mathbb{V}_{2}^{*}\left(\right.$ i.e., $\mathcal{A}_{2} \in \mathcal{L}\left(\mathbb{V}_{2}, \mathbb{V}_{2}^{*}\right)$ ) defined by

for any $\mathbf{B}, \mathbf{C} \in \mathbb{V}_{2}$.

$$
\left\langle\mathcal{A}_{2} \mathbf{B}, \mathbf{C}\right\rangle=S((\mathbf{B}, \mathbf{C}))_{2},
$$

For any $\mathbf{u} \in \mathbb{W}^{1, p}$ we set

$$
\mathcal{E}(u)=\frac{1}{2}\left[(\nabla \mathbf{u})+(\nabla \mathbf{u})^{T}\right] .
$$

Let us recall the following results whose proofs can be found in [36, Chapter 5, Theorem 1.10].

Lemma 2.2 (Korn's inequalities). Let $1<q<\infty$ and let $\mathcal{Q} \subset \mathbb{R}^{n}$ be of class $C^{1}$. Then there exist two positive constants $K_{q}^{i}=K_{q}^{i}(\mathcal{Q}), i=1,2$ such that

$$
K_{q}^{1}\|\mathbf{u}\|_{1, q} \leq\left(\int_{\mathcal{Q}}|\mathcal{E}(\mathbf{u})|^{q} d x\right)^{\frac{1}{q}} \leq K_{q}^{2}\|\mathbf{u}\|_{1, q},
$$

for any $\mathbf{u} \in \mathbb{V}_{1, q}$.

We introduce a nonlinear mapping $\mathcal{A}_{p}$ from $\mathbb{V}_{1, p}$ into $\mathbb{V}_{1, p}^{*}$ by setting

$$
\left\langle\mathcal{A}_{p} \mathbf{u}, \mathbf{v}\right\rangle=\int_{\mathcal{Q}} \mathbf{T}(\mathcal{E}(\mathbf{u})) \cdot \mathcal{E}(\mathbf{v}) d x
$$

for any $\mathbf{u}, \mathbf{v} \in \mathbb{V}_{1, p}$. Now we can define a nonlinear operator $\mathcal{A}$ from $\mathbb{V}$ into $\mathbb{V}^{*}$ by

$$
\langle\mathcal{A} \Phi, \Psi\rangle=\left\langle\mathcal{A}_{p} \mathbf{u}, \mathbf{v}\right\rangle+\left\langle\mathcal{A}_{2} \mathbf{B}, \mathbf{C}\right\rangle,
$$

for any $\Phi=(\mathbf{u} ; \mathbf{B}), \Psi=(\mathbf{v} ; \mathbf{C}) \in \mathbb{V}$. We state very important properties of $\mathcal{A}$ in the following

Lemma 2.3. Let $\mathbf{T}$ and $\Sigma$ satisfy (2)-(4) with $p \geq 2$. Then,

(1) the operator $\mathcal{A}$ is monotone; that is,

$$
\left\langle\mathcal{A} \Phi_{1}-\mathcal{A} \Phi_{2}, \Phi_{1}-\Phi_{2}\right\rangle \geq 0
$$

for any $\Phi_{1}, \Phi_{2} \in \mathbb{V}$. 
(2) There exists a constant $\tilde{\nu}$ such that

$$
\langle\mathcal{A} \Phi, \Phi\rangle \geq \tilde{\nu}\|\Phi\|_{\mathbb{V}}^{p, 2},
$$

for any $\Phi \in \mathbb{V}$.

(3) Also, there exists a positive constant $C$ such that

$$
\|\mathcal{A} \Phi\|_{\mathbb{V} *}^{p^{*}, 2} \leq C\left(1+\|\Phi\|_{\mathbb{V}}^{p, 2}\right),
$$

for any $\Phi \in \mathbb{V}$. Here

$$
\frac{1}{p}+\frac{1}{p^{*}}=1
$$

and

$$
\|\Psi\|_{\mathbb{V}^{*}}^{p^{*}, 2}=\left.\|\psi\|\right|_{\mathbb{V}_{2}^{*}} ^{2}+\|\phi\|_{\mathbb{V}_{1, p}^{*}}^{p^{*}},
$$

for $\Psi=(\phi ; \psi) \in \mathbb{V}^{*}$.

Proof. It is known from [37, Lemma 1.19, page 198] that for any $p \geq 2$ there exist positive constants $\nu_{i}, i=3,4,5$, such that for all $\mathbf{D}, \mathbf{E} \in \mathbb{R}_{\text {sym }}^{n \times n}$ :

$$
\begin{aligned}
\mathbf{T}(\mathbf{D}) \cdot \mathbf{D} & \geq \nu_{3}\left(1+|\mathbf{D}|^{p-2}\right)|\mathbf{D}|^{2}, \\
|\mathbf{T}(\mathbf{D})| & \leq \nu_{4}(1+|\mathbf{D}|)^{p-1}, \\
(\mathbf{T}(\mathbf{D})-\mathbf{T}(\mathbf{E})) \cdot(\mathbf{D}-\mathbf{E}) & \geq \nu_{5}|\mathbf{D}-\mathbf{E}|^{2} .
\end{aligned}
$$

Therefore, it follows from (17) that

$$
\begin{aligned}
\left\langle\mathcal{A}_{p} \mathbf{u}-\mathcal{A}_{p} \mathbf{v}, \mathbf{u}-\mathbf{v}\right\rangle & =\int_{\mathcal{Q}}[\mathbf{T}(\mathcal{E}(\mathbf{u}))-\mathbf{T}(\mathcal{E}(\mathbf{v}))] \cdot[\mathbf{u}-\mathbf{v}] d x \\
& \geq \nu_{5} \int_{\mathcal{Q}}|\mathcal{E}(\mathbf{u})-\mathcal{E}(\mathbf{v})|^{2} d x
\end{aligned}
$$

for any $\mathbf{u}, \mathbf{v} \in \mathbb{V}_{1, p}$. It is easily seen that

$$
\left\langle\mathcal{A}_{2} \mathbf{B}-\mathcal{A}_{2} \mathbf{C}, \mathbf{B}-\mathbf{C}\right\rangle \geq S\|\mathbf{B}-\mathbf{C}\|_{2}^{2},
$$

for any $\mathbf{B}, \mathbf{C} \in \mathbb{V}_{2}$. Therefore, it follows from (18)-(19) that $\mathcal{A}$ is monotone.

Now it follows from (15) that

$$
\begin{aligned}
\langle\mathcal{A} \Phi, \Phi\rangle & =\int_{\mathcal{Q}} \mathbf{T}(\mathcal{E}(\mathbf{u})) \cdot \mathcal{E}(\mathbf{u}) d x+S\|\mathbf{B}\|_{2}^{2}, \\
& \geq \nu_{3} \int_{\mathcal{Q}}\left(|\mathcal{E}(\mathbf{u})|^{2}+|\mathcal{E}(\mathbf{u})|^{p}\right) d x+S\|\mathbf{B}\|_{2}^{2},
\end{aligned}
$$

for any $\Phi \in \mathbb{V}$. Owing to Korn's inequalities we infer from the last estimate that

$$
\begin{aligned}
\langle\mathcal{A} \Phi, \Phi\rangle & \geq \tilde{\nu}_{3}\left(\|\mathbf{u}\|_{1}^{2}+\|\mathbf{u}\|_{1, p}^{p}\right)+S\|\mathbf{B}\|_{2}^{2}, \\
& \geq \tilde{\nu}_{3}\|\mathbf{u}\|_{1, p}^{p}+S\|\mathbf{B}\|_{2}^{2},
\end{aligned}
$$

which implies that there exists a constant $\tilde{\nu}$ such that (13) holds.

We have that

$$
\left\|\mathcal{A}_{2} \mathbf{B}\right\|_{\mathbb{V}_{2}^{*}}^{2} \leq S^{2}\|\mathbf{B}\|_{2}^{2}
$$

Also,

$$
\begin{aligned}
\left\|\mathcal{A}_{p} \mathbf{u}\right\|_{\mathbb{V}_{1, p}^{*}} & =\sup _{\|\mathbf{v}\|_{1, p}=1}\left|\left\langle\mathcal{A}_{p} \mathbf{u}, \mathbf{v}\right\rangle\right| \\
& \leq C \sup _{\|\mathbf{v}\|_{1, p}=1}\left(\int_{\mathcal{Q}}|\mathbf{T}(\mathcal{E}(\mathbf{u}))|^{p^{*}} d x\right)^{\frac{1}{p^{*}}}\left(\int_{\mathcal{Q}}|\mathcal{E}(\mathbf{v})|^{p} d x\right)^{\frac{1}{p}} .
\end{aligned}
$$


Thanks to Korn's inequalities and (16) we have

$$
\begin{aligned}
& \left\|\mathcal{A}_{p} \mathbf{u}\right\|_{\mathbb{V}_{1, p}^{*}} \leq C \sup _{\|\mathbf{v}\|_{1, p}=1}\left(\int_{\mathcal{Q}}|\mathbf{T}(\mathcal{E}(\mathbf{u}))|^{p^{*}} d x\right)^{\frac{1}{p^{*}}}\|\mathbf{v}\|_{1, p}, \\
& \left\|\mathcal{A}_{p} \mathbf{u}\right\|_{\mathbb{V}_{1, p}^{*}}^{p^{*}} \leq C \int_{\mathcal{Q}}(1+|\mathcal{E}(\mathbf{u})|)^{p} d x .
\end{aligned}
$$

By using Korn's inequalities into (21), we can deduce from the resulting estimate and (20) that (14) holds.

For any $p \geq 2$ and $\mathbf{u}, \mathbf{v}, \mathbf{w} \in \mathbb{W}^{1, p}$, we set

$$
b(\mathbf{u}, \mathbf{v}, \mathbf{w})=\int_{\mathcal{Q}} u_{i} \frac{\partial v_{j}}{\partial x_{i}} w_{j} d x
$$

where summations over repeated indices are enforced. The trilinear form $b(\mathbf{u}, \mathbf{v}, \mathbf{w})$ is continuous on $\mathbb{H}^{1}(\mathcal{Q}) \times \mathbb{H}^{1}(\mathcal{Q}) \times \mathbb{H}^{1}(\mathcal{Q})$. Moreover,

$$
\begin{aligned}
b(\mathbf{u}, \mathbf{v}, \mathbf{v}) & =0, \\
b(\mathbf{u}, \mathbf{v}, \mathbf{w}) & =-b(\mathbf{u}, \mathbf{w}, \mathbf{v}),
\end{aligned}
$$

for any $\mathbf{u} \in \mathbb{V}_{2}, \mathbf{v}, \mathbf{w} \in \mathbb{H}^{1}(\mathcal{Q})$. Since $\mathbb{V}_{1} \subset \mathbb{V}_{2}$ and $\mathbb{V}_{1, p} \subset \mathbb{V}_{2}$, then (23) and (24) are also valid for any element $\mathbf{u}$ in $\mathbb{V}_{1}$ and $\mathbb{V}_{1, p}$. For the proofs of the above properties and more information on the trilinear form $b(., .,$.$) , we refer, for instance, to [70, Chapter II, Subsection 1.2]. Set$

$$
\mathcal{B}_{0}\left(\Phi_{1}, \Phi_{2}, \Phi_{3}\right)=b\left(\mathbf{u}_{1}, \mathbf{u}_{2}, \mathbf{u}_{3}\right)-\mu b\left(\mathbf{B}_{1}, \mathbf{B}_{2}, \mathbf{u}_{3}\right)+\mu b\left(\mathbf{u}_{1}, \mathbf{B}_{2}, \mathbf{B}_{3}\right)-\mu b\left(\mathbf{B}_{1}, \mathbf{u}_{2}, \mathbf{B}_{3}\right),
$$

for any $\Phi_{i}=\left(\mathbf{u}_{i} ; \mathbf{B}_{i}\right) \in \mathbb{V}, i=1,2,3$. It follows from (23) and (24) that

$$
\begin{aligned}
& \mathcal{B}_{0}\left(\Phi_{1}, \Phi_{2}, \Phi_{2}\right)=0, \\
& \mathcal{B}_{0}\left(\Phi_{1}, \Phi_{2}, \Phi_{3}\right)=-\mathcal{B}_{0}\left(\Phi_{1}, \Phi_{3}, \Phi_{2}\right),
\end{aligned}
$$

for any $\Phi_{i} \in \mathbb{V}, i=1,2,3$. Following the idea in [35], we choose $s>1+\frac{n}{2}$ and set

$$
\begin{aligned}
\mathbb{W}_{1, s} & =\text { closure of } \mathcal{V}_{1} \text { in } \mathbb{H}^{s}(\mathcal{Q}), \\
& =\left\{\mathbf{u} \in \mathbb{H}_{0}^{s}, \operatorname{div} \mathbf{u}=0\right\} \\
\mathbb{W}_{2, s} & =\text { closure of } \mathcal{V}_{2} \text { in } \mathbb{H}^{s}(\mathcal{Q}),
\end{aligned}
$$

and

$$
\mathbb{W}_{s}=\mathbb{W}_{1, s} \times \mathbb{W}_{2, s} .
$$

The spaces $\mathbb{W}_{i, s}, i=1,2$ will be equipped with the usual scalar product and norm of $\mathbb{H}^{s}(\mathcal{Q})$ respectively denoted by $((\cdot, \cdot))_{s}$ and $\|\cdot\|_{s}$. We also use these symbols to denote the norm and scalar product of $\mathbb{W}_{s}$. Identifying $\mathbb{H}$ with its dual, we have the following Gelfand chain

$$
\mathbb{W}_{s} \subset \mathbb{V} \subset \mathbb{H} \subset \mathbb{V}^{*} \subset \mathbb{W}_{s}^{*}
$$

where each space is densely and compactly embedded into the next one.

Since $s-1>n / 2, \partial \Phi / \partial x_{i}$ is an element of $\mathbb{L}^{\infty}(\mathcal{Q})$ for any $\Phi_{3} \in \mathbb{W}_{s}$. Therefore

$$
\begin{aligned}
\left|\mathcal{B}_{0}\left(\Phi_{1}, \Phi_{2}, \Phi_{3}\right)\right|=\mid & -\mathcal{B}_{0}\left(\Phi_{1}, \Phi_{3}, \Phi_{2}\right) \mid, \\
& \leq C\left|\Phi_{1}\right|\left|\Phi_{2}\right||| \Phi \|_{\mathbb{W}_{s}},
\end{aligned}
$$

for any $\Phi_{1}, \Phi_{2} \in \mathbb{V}$ and $\Phi_{3} \in \mathbb{W}_{s}$. From this we infer the existence of a continuous bilinear form $\mathcal{B}(\cdot, \cdot)$ defined on $\mathbb{V} \times \mathbb{V}$ taking its values in $\mathbb{W}_{s}^{*}$. This bilinear mapping satisfies the properties stated in the following 
Lemma 2.4. (i) For any $\Phi_{1}, \Phi_{2} \in \mathbb{V}$ and $\Phi_{3} \in \mathbb{W}_{s}$,

$$
\left\langle\mathcal{B}\left(\Phi_{1}, \Phi_{2}\right), \Phi_{3}\right\rangle=\mathcal{B}_{0}\left(\Phi_{1}, \Phi_{2}, \Phi_{3}\right)
$$

(ii) We have that

$$
\left\langle\mathcal{B}\left(\Phi_{1}, \Phi_{2}\right), \Phi_{2}\right\rangle=0
$$

for any $\Phi_{1} \in \mathbb{V}$ and $\Phi_{2} \in \mathbb{W}_{s}$.

(iii) There exists a positive constant $C$ such that

$$
\left\|\mathcal{B}\left(\Phi_{1}, \Phi_{2}\right)\right\|_{\mathbb{W}_{s}^{*}} \leq C\left|\Phi_{1} \| \Phi_{2}\right|
$$

for any $\Phi_{i} \in \mathbb{V}, i=1,2$.

Proof. All of the statements in the lemma were proved above (see the lines between (26)-(31)).

2.2. Stochastic setting and some hypotheses. Let $T>0$ and $(\Omega, \mathcal{F}, \mathbb{P})$ be a complete probability space. We endow the probability space $(\Omega, \mathcal{F}, \mathbb{P})$ with an increasing filtration $(\mathbb{F}=$ $\left.\mathcal{F}_{t}\right)_{t \in[0, T]}$. The probability space satisfies the usual condition, that is :

(1) $\mathbb{P}$ is complete in $(\Omega, \mathcal{F})$,

(2) $\mathcal{F}_{0}$ contains all null sets of $(\Omega, \mathcal{F}, \mathbb{P})$,

(3) the filtration $\mathbb{F}$ is right-continuous.

The following definition is borrowed from [12, Definition 4.1]

Definition 2.5. Let $(\Omega, \mathcal{F}, \mathbb{P}, \mathbb{F})$ be a filtered probability space and $\mathbb{K}$ be a real separable Hilbert space. An $\mathbb{F}$-adapted cylindrical Wiener process on $\mathbb{K}$ is a family $\mathbf{W}=(\mathbf{W}(t))_{t \in[0, T]}$ of bounded linear operators from $\mathbb{K}$ into $L^{2}(\Omega, \mathcal{F}, \mathbb{P})^{1}$ such that

(1) for all $t \geq 0$, and $\mathbf{k}_{1}, \mathbf{k}_{2} \in \mathbb{K}, \mathbb{E}\left[\mathbf{W}(t) \mathbf{k}_{1} \mathbf{W}(t) \mathbf{k}_{2}\right]=\left\langle\mathbf{k}_{1}, \mathbf{k}_{2}\right\rangle_{\mathbb{K}}$,

(2) for each $\mathbf{k} \in \mathbb{K}, t \geq 0, \mathbf{W}(t) \mathbf{k}$ is a real valued $\mathbf{F}$-adapted Wiener process.

For real separable Banach spaces $\mathbb{U}$ and $H$ we denote by $L(\mathbb{U}, H)$ the space of continuous linear mapping from $\mathbb{U}$ into $H$. By $J_{2}(\mathbb{U}, H)$ we mean the subspace of $L(\mathbb{U}, H)$ consisting of HilbertSchmidt operators when $\mathbb{U}$ and $H$ are separable. In what follows we set $J_{2}(\mathbb{U}):=J_{2}(\mathbb{U}, \mathbb{U})$. It is known that $J_{2}(\mathbb{U}, H)$ is a Hilbert space and its norm is denoted by $\|S\|_{J_{2}}$ for any $S \in J_{2}(\mathbb{U}, H)$. Following [16, Proposition 4.11] (see also [12, Remark 4.2]) we can represent a cylindrical Wiener process on $\mathbb{K}$ as a formal series

$$
\mathbf{W}(t)=\sum_{i=1}^{\infty} w_{i}(t) \varphi_{i}(t), t \geq 0
$$

where $\left(w_{i}\right)_{i \in \mathbb{N}}$ is a family of independent standard 1-dimensional Wiener processes, and $\left(\varphi_{i}\right)_{i \in \mathbb{N}}$ is an orthonormal basis of $\mathbb{K}$. The above series does not satisfy any notion of convergence in the Hilbert space $\mathbb{K}$ but it does on $L^{2}(\Omega, \mathcal{F}, \mathbb{P} ; C(0, T ; \mathbb{U}))$ for any Hilbert space $\mathbb{U}$ such that the embedding $\mathbb{K} \subset \mathbb{U}$ is Hilbert-Schmidt. The series admits an $\mathbb{U}$-valued continuous modification $\mathbb{P}$-almost surely.

Next, we recall the definition of a $Q$ Wiener process by following [54] and [71]. For this let $\mathbb{U}$ be a separable Banach space and $Q: \mathbb{U}^{*} \rightarrow \mathbb{U}$ such that

$$
\begin{aligned}
& \left\langle Q u^{*}, u^{*}\right\rangle \geq 0, \\
& \left\langle Q u^{*}, v^{*}\right\rangle=\left\langle u^{*}, Q v^{*}\right\rangle,
\end{aligned}
$$

for any $u^{*}, v^{*} \in \mathbb{U}^{*}$. The range $\operatorname{Ran}(Q)$ of $Q$ is a pre-Hilbert space when equipped with the scalar product $[\cdot, \cdot]_{Q}$ defined by

$$
\left[Q u^{*}, Q v^{*}\right]_{Q}=\left\langle Q u^{*}, v^{*}\right\rangle, \forall u^{*}, v^{*} \in \operatorname{Ran}(Q) .
$$

\footnotetext{
${ }^{1}$ The space of real-valued square integrable random variables defined on $(\Omega, \mathcal{F}, \mathbb{P})$ is denoted by $L^{2}(\Omega, \mathcal{F}, \mathbb{P})$
} 
Let $H_{Q}$ be the completion of $\operatorname{Ran}(Q)$ with respect to $[\cdot, \cdot]_{Q}$. It is called the Reproducing Kernel Hilbert Space (RKHS) associated with $Q$. Let $i_{Q}^{*}$ be the dual operator of the injection mapping $i_{Q}: H_{Q} \rightarrow \mathbb{U}$. The operator $Q$ admits the decomposition $Q=i_{Q} i_{Q}^{*}$.

Definition 2.6. Let $\mathbb{U}$ be a separable Banach space and $Q: \mathbb{U}^{*} \rightarrow \mathbb{U}$ be a symmetric and nonnegative operator as above. An $\mathbb{F}$-adapted stochastic process $\mathbf{W}$ is a $Q$-Wiener process taking values in $\mathbb{U}$ if

(1) $i_{Q}: H_{Q} \rightarrow \mathbb{U}$ is $\gamma$-Radonifying operator,

(2) $\mathbf{W}(0)=0$,

(3) $\mathbf{W}$ has continuous trajectories,

(4) W has independent increments,

(5) for any $0 \leq s \leq t$ the random variable $\mathbb{Y}=\mathbf{W}(t)-\mathbf{W}(s)$ is Gaussian with zero mean and covariance $(t-s) Q$, that is, its characteristic function is of the form

$$
\begin{aligned}
\varphi_{\mathbb{Y}}\left(u^{*}\right) & =\mathbb{E}\left(\exp \left[i \mathbb{Y} u^{*}\right]\right), \\
& =\exp \left(-\frac{1}{2}[t-s]\left\langle Q u^{*}, u^{*}\right\rangle\right), u^{*} \in \mathbb{U}^{*} .
\end{aligned}
$$

We refer, for instance, to [46] and [7] and references therein for more information about $\gamma$ Radonifying operators and their use in the context of stochastic calculus.

Remark 2.7. When $Q=I d$ and $\mathbb{U}$ is a separable Hilbert space we recover from the above definition the concept of cylindrical Wiener process on $\mathbb{U}$, see [54] for more details.

Remark 2.8. Let $\mathbb{U}$ and $\mathbb{K}$ be such two real separable Hilbert spaces that the canonical injection $\mathcal{J}$ from $\mathbb{K}$ into $\mathbb{U}$ is Hilbert-Schmidt. Let us denote by $\mathcal{J}^{*}$ the adjoint of $\mathcal{J}$. It is easy to see that $Q=\mathcal{J J}^{*}$ is a symmetric and nonnegative operator with $\operatorname{tr} Q<\infty$. Thanks to [16, Proposition 4.11] we can view the cylindrical Wiener process $\mathbf{W}$ on $\mathbb{K}$ defined by (35) as a $Q$-Wiener process with values in $\mathbb{U}$ and

$$
Q^{\frac{1}{2}}(\mathbb{U})=\mathbb{K}
$$

Conversely, let $\mathbf{W}$ be a $\mathcal{J J}^{*}$-Wiener processor with values in $\mathbb{U}$. Let $\left\{\zeta_{j}: j \in \mathbb{N}\right\}$ be an orthonormal basis of $\mathbb{U}$ consisting of eigenfunctions of $\mathcal{J J}^{*}$; that is, there exists an increasing family $\left\{\lambda_{j}: j \in \mathbb{N}\right\}$ of positive numbers such that $\mathcal{J J}^{*} \zeta_{j}=\lambda_{j} \zeta_{j}$. Using the definition of $\left\{\zeta_{j} ; j \in \mathbb{N}\right\}$ we can easily check that $\left\{\varphi_{j}=\frac{\mathcal{J}^{*} \zeta_{j}}{\sqrt{\lambda_{j}}}: j \in \mathbb{N}\right\}$ forms an orthonormal basis of $\mathbb{K}$. Thanks to [16, Theorem 4.3] $\mathbf{W}$ can be written as a formal series

$$
\mathbf{W}(t)=\sum_{j=1}^{\infty} w_{j}(t) \frac{\mathcal{J}^{*} \zeta_{j}}{\sqrt{\lambda_{j}}},
$$

where $\left\{w_{j}(t)=\sqrt{\lambda_{j}}\left\langle\mathbf{W}(t), \zeta_{j}\right\rangle_{\mathbb{U}} ; j \in \mathbb{N}\right\}$ is a sequence of independent real-valued standard Wiener processes. Hence $\mathbf{W}$ defines a cylindrical Wiener process on $\mathbb{K}$.

Now let $\mathbb{K}_{i}, i=1,2$ be two fixed separable Hilbert spaces such that the canonical injections $\mathcal{I}_{i}$ from $\mathbb{H}_{i}$ into $\mathbb{K}_{i}$, for $i=1,2$, are Hilbert-Schmidt. For $i=1,2$ let $\left\{\tilde{e}_{k, i}: k \geq 1\right\}$ be the orthonormal bases of $\mathbb{K}_{i}$ consisting of the eigenfunctions of $\tilde{Q}_{i}=\mathcal{I}_{i} \mathcal{I}_{i}^{*}$. The corresponding eigenvalues of $\left\{\tilde{e}_{k, i}: k \geq 1\right\}$ are $\left\{\tilde{\lambda}_{k, i}: k \in \mathbb{N} \cap[1, \infty)\right\}, i=1,2$. Throughout we denote by $\left\{e_{k, i}:\right.$ $k \in \mathbb{N} \cap[1, \infty)\}$ another orthonormal basis of $\mathbb{H}_{i}$ such that $e_{k, i}=\frac{\mathcal{I}_{i}^{*} \tilde{e}_{k, i}}{\sqrt{\tilde{\lambda}_{k, i}}}$ for each $k \in \mathbb{N} \cap[1, \infty)$ and $i=1,2$. Let $\mathcal{W}=(\mathcal{W}(t))_{t \in[0, T]}$ and $\mathbf{W}=(\mathbf{W}(t))_{t \in[0, T]}$ be two mutually independent cylindrical Wiener processes on $\mathbb{H}_{1}$ and $\mathbb{H}_{2}$ respectively. We represent $\mathcal{W}$ and $\mathbf{W}$ as two formal series $\mathcal{W}(t)=\sum_{k=1}^{\infty} \mathcal{W}_{k} e_{k, 1}$ and $\mathbf{W}(t)=\sum_{k=1}^{\infty} \mathbf{W}_{k} e_{k, 2}$ where $\left\{W^{k}=\left(\mathcal{W}_{k} ; \mathbf{W}_{k}\right): k=1,2, \ldots\right\}$ is 
a sequence of mutually independent standard $\mathbb{R}^{2}$-valued Brownian motion. Now we denote by $\mathbb{K}$ the cartesian product $\mathbb{K}_{1} \times \mathbb{K}_{2}$ and set

$$
W=\left(\begin{array}{l}
\mathcal{W} \\
\mathbf{W}
\end{array}\right)
$$

and

$$
J_{2}(\mathbb{K}, \mathbb{H})=J_{2}\left(\mathbb{K}_{1}, \mathbb{H}_{1}\right) \times J_{2}\left(\mathbb{K}_{2}, \mathbb{H}_{2}\right) .
$$

A new orthonormal basis of $\mathbb{H}$ is $\left\{e_{k}=\left(e_{k, 1} ; e_{k, 2}\right) ; k \geq 1\right\}$ and the space $J_{2}(\mathbb{K}, \mathbb{H})$ is a Hilbert space endowed with the natural scalar product of the cartesian product whose corresponding norm is

$$
\|R\|_{J_{2}}=\left(\sum_{i=1}^{2}\left\|R_{i}\right\|_{J_{2, i}}^{2}\right)^{\frac{1}{2}},
$$

where $J_{2, i}=J_{2}\left(\mathbb{K}_{i}, \mathbb{H}_{i}\right)$ for $i=1,2$. The stochastic processes $W$ defines a cylindrical process on $\mathbb{H}$. By Remark 2.8 it can be considered as a $Q$-Wiener process taking values in $\mathbb{K}$ such that $W \in C(0, T ; \mathbb{K}) \mathbb{P}$-almost surely. Here $Q=\mathcal{I I}^{*}$ where $\mathcal{I}$ is the canonical injection obtained from the Hilbert-Schmidt embedding $\mathbb{H} \subset \mathbb{K}$. In terms of $\mathcal{I}_{i}$ and $\tilde{Q}_{i}$, we have

$$
I=\left(\begin{array}{cc}
\mathcal{I}_{1} & 0 \\
0 & \mathcal{I}_{2}
\end{array}\right) \text { and } Q=\left(\begin{array}{cc}
\tilde{Q}_{1} & 0 \\
0 & \tilde{Q}_{2}
\end{array}\right)
$$

Thanks to Remark 2.8 we have $Q^{\frac{1}{2}}(\mathbb{K})=\mathbb{H}$. Furthermore, the cylindrical Wiener process $W$ admits the series representation

$$
W=\sum_{k=1}^{\infty} W^{k} \cdot e_{k}
$$

Let $X_{1}^{*}=\mathbb{V}_{1, p}^{*}$ and $X_{2}^{*}=\mathbb{V}_{2}^{*}$. Now we introduce the hypotheses on $f_{i}(\mathbf{u}, \mathbf{B}, t), g_{i}(\mathbf{u}, \mathbf{B}, t)$ that are relevant for the major part of the paper.

(F) We assume that $f_{i}: \mathbb{H} \times[0, T] \rightarrow \mathbb{X}_{i}^{*}(i=1,2)$ are nonlinear mappings such that

(a) they are continuous with respect to $t$,

(b) $(\mathbf{u} ; \mathbf{B}) \mapsto f_{i}(\mathbf{u}, \mathbf{B}, t)$ are continuous for all $t \in[0, T]$; there exists a positive constant $C$ such that

$$
\left\|f_{i}(\mathbf{u}, \mathbf{B}, t)\right\|_{X_{i}^{*}} \leq C(1+|\mathbf{u}|+|\mathbf{B}|),
$$

for any $(\mathbf{u} ; \mathbf{B}) \in \mathbb{H}$ and $t \in[0, T]$.

(G) We suppose that $g_{i}: \mathbb{H} \times[0, T] \rightarrow J_{2}\left(\mathbb{H}_{i}\right)$ are nonlinear mappings such that

(a) the $g_{i}$-s are continuous,

(b) there exists a positive constant $C$ such that

$$
\left\|g_{i}(\mathbf{u}, \mathbf{B}, t)\right\|_{J_{2, i}} \leq C(1+|\mathbf{u}|+|\mathbf{B}|),
$$

for any $(\mathbf{u} ; \mathbf{B}) \in \mathbb{H}$ and $t \in[0, T]$.

Now we introduce the operators

$$
f=\left(\begin{array}{cc}
f_{1} & 0 \\
0 & f_{2}
\end{array}\right): \mathbb{H}_{1} \times \mathbb{H}_{2} \times[0, T] \rightarrow \mathbb{V}^{*} .
$$

and

$$
g=\left(\begin{array}{cc}
g_{1} & 0 \\
0 & g_{2}
\end{array}\right): \mathbb{H}_{1} \times \mathbb{H}_{2} \times[0, T] \rightarrow J_{2}(\mathbb{H}) .
$$


Then, modulo divergence freeness, the problem (1) can be rewritten as

$$
\begin{aligned}
& d \mathbf{y}+[\mathcal{A} \mathbf{y}+\mathcal{B}(\mathbf{y}, \mathbf{y})] d t=f(\mathbf{y}, t) d t+g(\mathbf{y}, t) d W \\
& \mathbf{y}(0)=\mathbf{y}_{0} \in \mathbb{H},
\end{aligned}
$$

where $\mathbf{y}=(\mathbf{u} ; \mathbf{B})$ is a solution of $(1)$ and $\mathbf{y}_{0}=\left(\mathbf{u}_{0} ; \mathbf{B}_{0}\right)$. From now on, we will work with (38)-(39).

Remark 2.9. Let $W$ be a Wiener process taking values in a separable Hilbert space $\mathbb{U}$ and with covariance operator $\tilde{Q}$ such that $\operatorname{tr} \tilde{Q}<\infty$ and let $H$ another separable Hilbert space. From [16] the stochastic integral $\int_{0}^{T} \Psi(t) d W(t)$ is well defined for any progressively measurable process $\Psi$ taking values in $J_{2}(\tilde{Q}(\mathbb{U}), H)$ such that

$$
\mathbb{E} \int_{0}^{T}\|\Psi(t)\|_{J_{2}(\tilde{Q}(\mathbb{U}), H)}^{2} d t<\infty .
$$

In our framework, since $Q^{\frac{1}{2}}(\mathbb{K})=\mathbb{H}$ (see Remark 2.8) the stochastic integral is well defined as long as $\Psi$ is progressively measurable, takes values in $J_{2}(\mathbb{H})$ and satisfies

$$
\mathbb{E} \int_{0}^{T}\|\Psi(t)\|_{J_{2}(\mathbb{H})}^{2} d t<\infty
$$

To close this section let us introduce additional notations frequently used throughout the work. The mathematical expectation with respect to the probability measure $\mathbb{P}$ is denoted by $\mathbb{E}$. Let $r \in[2, \infty)$ and following [17, Section 3.1] $L^{r / 2}\left(\Omega, L^{p, 2}(0, T ; \mathbb{V})\right)$ the space of functions $\mathbf{y}=\mathbf{y}(\omega, t)$ defined on $\Omega \times[0, T]$ with values in $\mathbb{V}$ such that:

(a) $\mathbf{y}(\cdot, \cdot)$ is $\mathcal{F} \times \mathcal{B}([0, T])$-measurable,

(b) $\mathbf{y}(\omega, t) \in \mathbb{V}$ for almost all $(\omega, t)$ and

$$
\mathbb{E}\left(\int_{0}^{T}\|\mathbf{y}(s)\|_{\mathbb{V}}^{p, 2} d s\right)^{\frac{r}{2}}<C .
$$

We also set

$$
L^{2}\left(\Omega, L^{p, 2}(0, T ; \mathbb{V})\right)=L^{2}\left(\Omega, L^{p}\left(0, T ; \mathbb{V}_{1, p}\right)\right) \times L^{2}\left(\Omega, L^{2}\left(0, T ; \mathbb{V}_{2}\right)\right),
$$

and

$$
L^{2}\left(\Omega, L^{p^{*}, 2}\left(0, T ; \mathbb{V}^{*}\right)\right)=L^{2}\left(\Omega, L^{p^{*}}\left(0, T ; \mathbb{V}_{1, p}^{*}\right)\right) \times L^{2}\left(\Omega, L^{2}\left(0, T ; \mathbb{V}_{2}^{*}\right)\right),
$$

where $p^{*}=\frac{p}{p-1}$.

\section{EXISTENCE OF MARTINGALE WEAK SOLUTION}

In this section we state our first main result and give its proof. Before we proceed further we explicitly define what we mean by a (martingale) weak solution of (38)-(39).

Definition 3.1. A weak solution of (38)-(39) is a system $\left(\Omega, \mathbb{F},\left\{\mathbb{F}^{t}: t \geq 0\right\}, \mathbb{P}, W, \mathbf{y}\right)$ where

(i) $(\Omega, \mathbb{F}, \mathbb{P})$ is a probability space, $\left\{\mathbb{F}^{t}: t \geq 0\right\}$ is an increasing filtration satisfying the usual conditions,

(ii) $W$ is a cylindrical Wiener process wrt $\left\{\mathbb{F}^{t}: t \geq 0\right\}$,

(iii) $\mathbf{y}$ is an element of $L^{r / 2}\left(\Omega, L^{p, 2}(0, T ; \mathbb{V})\right)$ for any $r \in[2, \infty)$,

(iv) the stochastic process $\mathbf{y}$ is predictable, 
(v) for any $\mathbf{w} \in \mathbb{W}_{s}$,

$$
\begin{aligned}
(\mathbf{y}(t), \mathbf{w})+\int_{0}^{t}(\langle\mathcal{A} \mathbf{y}(s)+\mathcal{B}(\mathbf{y}(s), \mathbf{y}(s)), \mathbf{w}\rangle) d s & =\left(\mathbf{y}_{0}, \mathbf{w}\right)+\int_{0}^{t}\langle f(\mathbf{y}(s), s), \mathbf{w}\rangle d s \\
& +\int_{0}^{t}(g(\mathbf{y}(s), s), \mathbf{w}) d W
\end{aligned}
$$

almost surely and for all $t \in[0, T]$.

(vi) The function $\mathbf{y}$ belongs to $L^{r}(\Omega, C(0, T ; \mathbb{H})), r \in[2, \infty)$.

Before we proceed further we make the following remark.

Remark 3.2. We should note that we use the notation

$$
\int_{0}^{t}(g(\mathbf{y}(s), s), \mathbf{w}) d W=\sum_{k=1}^{\infty} \int_{0}^{t}\left(g(\mathbf{y}(s), s) e_{k}, \mathbf{w}\right) d W^{k} .
$$

Our first main result is stated in the following theorem.

Theorem 3.3. Let $p_{\min }=\max \left(n, 1+\frac{2 n}{n+2}, \frac{n+2}{4}\right)$. Let the conditions $(2)$-(4) hold with $p \geq p_{\min }$. Let $(F)$, and $(G)$ be satisfied and $\mathbf{y}_{0} \in \mathbb{H}$. Then there exists at least a weak solution of (38)-(39) in the sense of Definition 3.1.

The proof of this statement is given in the next section and it relies very much on Galerkin, compactness and monotonicity methods.

3.1. Galerkin approximation and a priori estimates. In this subsection we introduce the Galerkin approximation scheme of our problem and derive a priori estimates for the solution.

We introduce the family of eigenfunctions

$$
\left(\left(\mathbf{w}_{j}, \mathbf{v}\right)\right)_{s}=\lambda_{j}^{1}\left(\mathbf{w}_{j}, \mathbf{v}\right), \forall \mathbf{v} \in \mathbb{W}_{1, s}
$$

and

$$
\left(\left(\mathbf{C}_{j}, \mathbf{B}\right)\right)_{s}=\lambda_{j}^{2}\left(\mathbf{C}_{j}, \mathbf{B}\right), \forall \mathbf{B} \in \mathbb{W}_{2, s} .
$$

Then, we can define a spectral problem on $\mathbb{W}_{s}$ by setting

$$
\Psi_{j}=\left(\Psi_{j}^{1} ; \Psi_{j}^{2}\right), \Psi_{j}^{1}=\mathbf{w}_{j}, \Psi_{j}^{2}=\mathbf{C}_{j},
$$

and

$$
\left(\left(\Psi_{j}, \mathbf{w}\right)\right)_{s}=\sum_{k=1}^{2} \lambda_{j}^{k}\left(\Psi_{j}^{k}, \mathbf{w}^{k}\right), \forall \mathbf{w}:=\left(\mathbf{w}^{1}, \mathbf{w}^{2}\right) \in \mathbb{W}_{s} .
$$

The last identity will be written in the following abstract form

$$
\left(\left(\Psi_{j}, \mathbf{w}\right)\right)_{s}=\lambda_{j}\left(\Psi_{j}, \mathbf{w}\right), \forall \mathbf{w} \in \mathbb{W}_{s}
$$

We assume that $\Psi_{j}, j=1,2,3, \cdots$ form an orthonormal basis of $\mathbb{W}_{s}$ which is complete in $\mathbb{V}$ and form an orthogonal basis of $\mathbb{H}$. Let $(\bar{\Omega}, \overline{\mathbb{F}}, \overline{\mathbb{P}}, \bar{W}),\left(\bar{W}=\sum_{i=1}^{\infty} \bar{W}^{i} \cdot e_{i}\right.$ is a cylindrical Wiener process evolving on $\mathbb{H}$ ) and $m$ a positive integer. We equip the probability space $(\bar{\Omega}, \overline{\mathbb{F}}, \overline{\mathbb{P}})$ with the natural filtration of $\bar{W}$ which is denoted by $\overline{\mathbb{F}}^{t}$. We set

$$
\mathbb{W}_{s}^{m}=\operatorname{Span}\left\{\Psi_{j}: j=1,2, \ldots, m\right\} .
$$

We look for a sequence of stochastic processes $\left(\mathbf{y}^{m}: m=1,2, \ldots\right) \subset \mathbb{W}_{s}^{m}$ such that

$$
\begin{aligned}
d\left(\mathbf{y}^{m}(s), \Psi_{j}\right)+\left\langle\mathcal{A} \mathbf{y}^{m}(s)+\mathcal{B}\left(\mathbf{y}^{m}(s), \mathbf{y}^{m}(s)\right), \Psi_{j}\right\rangle d s & =\left\langle f\left(\mathbf{y}^{m}(s), s\right), \Psi_{j}\right\rangle d s \\
& +\sum_{i=1}^{m}\left(g\left(\mathbf{y}^{m}(s), s\right) e_{i}, \Psi_{j}\right) d \bar{W}^{i},
\end{aligned}
$$




$$
\begin{gathered}
\mathbf{y}^{m}(0)=\mathbf{y}_{0}^{m} \in \mathbb{W}_{s}^{m}, \\
\mathbf{y}_{0}^{m} \rightarrow \mathbf{y}_{0} \text { in } \mathbb{H} \text { as } m \rightarrow \infty .
\end{gathered}
$$

Note that,

$$
\begin{aligned}
\mathbf{y}^{m}(t) & =\sum_{j=1}^{m}\left(\left(\mathbf{y}^{m}(t), \Psi_{j}\right)\right)_{s} \Psi_{j} \\
& \left.=\sum_{j=1}^{m} \lambda_{j}\left(\mathbf{y}^{m}(t), \Psi_{j}\right)\right] \Psi_{j}:=\sum_{j=1}^{m}\left[\sum_{k=1}^{2} \lambda_{j}^{k}\left(\mathbf{y}^{m}(t), \Psi_{j}^{k}\right)\right] \Psi_{j} .
\end{aligned}
$$

Thanks Lemma 2.4 and Assumptions (F) and (G) the nonlinear maps $\mathcal{B}, f$, and $g$ are continuous and locally bounded. Thanks to the fact that the potential $\Sigma$ is of class $C^{2} \mathcal{A}: \mathbf{V} \mapsto \mathbf{V}_{s}^{*}$ is hemicontinuous and monotone by Lemma 2.3. Therefore, we infer from [35, Chapitre II, page $171]$ and Lemma 2.3 that $\mathcal{A}$ is continuous from $\mathbb{V}$ into $\mathbb{V}^{*}$ and locally bounded. It then follows that the system (42)-(44) is a system of stochastic differential equations in a finite dimensional Banach space with continuous and locally bounded coefficients. From the proof of existence theorem in [65, Chapter 3, Section 3, page 59] (see also [27, Chapter IV, Section 2, pp 167-177]), which do not require the Lipschitz condition on the coefficients, there exists on a short interval $\left[0, T_{m}\right]$ a sequence of continuous functions $\mathbf{y}^{m}$ solving the system (42)-(44). It will follow from $a$ priori estimates that $\mathbf{y}^{m}$ exists on $[0, T]$.

First we prove the following lemma.

Lemma 3.4. The sequence $\left(\mathbf{y}^{m}: m=1,2, \ldots\right)$ satisfies

$$
\overline{\mathbb{E}} \sup _{s \in[0, T]}\left|\mathbf{y}^{m}(s)\right|^{r}<C,
$$

and

$$
\overline{\mathbb{E}}\left(\int_{0}^{T}\left\|\mathbf{y}^{m}(s)\right\|_{\mathbb{V}}^{p, 2} d s\right)^{\frac{r}{2}}<C,
$$

for any $r \in[2, \infty)$.

Proof. Let $M$ be a positive integer. We define a sequence of stopping times $\tau_{M}$ by setting

$$
\tau_{M}=\inf \left\{s:\left|\mathbf{y}^{m}(s)\right|+\left(\int_{0}^{s}\left\|\mathbf{y}^{m}(t)\right\|_{\mathbb{V}}^{p, 2} d t\right)^{\frac{1}{2}} \geq M\right\} \wedge T .
$$

We shall use a modification of the argument used in [1]. Let $t \in\left[0, T \wedge \tau_{M}\right]$. By Itô's formula, we have

$$
\begin{aligned}
\left|\mathbf{y}^{m}(t)\right|^{2}+2 \int_{0}^{t}\left\langle\mathcal{A} \mathbf{y}^{m}(s), \mathbf{y}^{m}(s)\right\rangle d s & =\left|\mathbf{y}_{0}^{m}\right|^{2}+2 \int_{0}^{t}\left\langle f\left(\mathbf{y}^{m}(s), s\right), \mathbf{y}^{m}(s)\right\rangle d s \\
& +\sum_{j, i=1}^{m} \sum_{k=1}^{2} \int_{0}^{t} \lambda_{j}^{k}\left(g\left(\mathbf{y}^{m}(s), s\right) e_{i}, \Psi_{j}^{k}\right)^{2} d s \\
& +2 \sum_{i=1}^{m} \int_{0}^{t}\left(g\left(\mathbf{y}^{m}(s), s\right) e_{i}, \mathbf{y}^{m}(s)\right) d \bar{W}^{i}
\end{aligned}
$$

Since

$$
\sum_{j, i=1}^{m} \sum_{k=1}^{2} \lambda_{j}^{k}\left(g\left(\mathbf{y}^{m}(s), s\right) e_{i}, \Psi_{j}^{k}\right)^{2} \leq\left\|g\left(\mathbf{y}^{m}(s), s\right)\right\|_{J_{2}}^{2},
$$


it follows from (13) and (49) that

$$
\begin{aligned}
\left|\mathbf{y}^{m}(t)\right|^{2}+2 \tilde{\nu} \int_{0}^{t}\left\|\mathbf{y}^{m}(s)\right\|_{\mathbb{V}}^{p, 2} d s & \leq\left|\mathbf{y}_{0}\right|^{2}+2 \int_{0}^{t}\left\|f\left(\mathbf{y}^{m}(s), s\right)\right\|\left\|_{\mathbb{V} *}|| \mathbf{y}^{m}(s)\right\|_{\mathbb{V}} d s \\
& +\int_{0}^{t}\left\|g\left(\mathbf{y}^{m}(s), s\right)\right\|_{J_{2}}^{2} d s \\
& +2 \sum_{i=1}^{m} \int_{0}^{t}\left(g\left(\mathbf{y}^{m}(s), s\right) e_{i}, \mathbf{y}^{m}(s)\right) d \bar{W}^{i} .
\end{aligned}
$$

For any $\varepsilon>0$, we easily check that

$$
\left\|f\left(\mathbf{y}^{m}(s), s\right)\right\|_{\mathbb{V}^{*}}\left\|\mathbf{y}^{m}(s)\right\|_{\mathbb{V}} \leq C_{\varepsilon}\left\|f\left(\mathbf{y}^{m}(s), s\right)\right\|_{\mathbb{V}^{*}}^{2}+\varepsilon\left\|\mathbf{y}^{m}(s)\right\|_{\mathbb{V}}^{p, 2}+C(\varepsilon, p) .
$$

Hence, owing to the assumptions on $f$ and $g$, we can derive from the last estimate and (50) that

$$
\begin{aligned}
\left|\mathbf{y}^{m}(t)\right|^{2}+\tilde{\nu} \int_{0}^{t}|| \mathbf{y}^{m}(s) \|_{\mathbb{V}}^{p, 2} d s \leq & \left|\mathbf{y}_{0}\right|^{2}+C \int_{0}^{t}\left(1+\left|\mathbf{y}^{m}(s)\right|^{2}\right) d s \\
& +2 \sum_{i=1}^{m} \int_{0}^{t}\left(g\left(\mathbf{y}^{m}(s), s\right) e_{i}, \mathbf{y}^{m}(s)\right) d \bar{W}^{i} .
\end{aligned}
$$

In view of $(52)$ we have

$$
\begin{aligned}
\overline{\mathbb{E}} \sup _{s \in\left[0, t \wedge \tau_{M}\right]}\left|\mathbf{y}^{m}(s)\right|^{2}+\tilde{\nu} \overline{\mathbb{E}} \int_{0}^{t \wedge \tau_{m}}\left\|\mathbf{y}^{m}(s)\right\|_{\mathbb{V}}^{p, 2} d s & \leq\left|\mathbf{y}_{0}\right|^{2}+C \overline{\mathbb{E}} \int_{0}^{t \wedge \tau_{M}}\left(1+\left|\mathbf{y}^{m}(s)\right|^{2}\right) d s \\
& +2 \overline{\mathbb{E}} \sup _{s \in\left[0, t \wedge \tau_{M}\right]}\left|\sum_{i=1}^{m} \int_{0}^{s}\left(g\left(\mathbf{y}^{m}(\tau), \tau\right) e_{i}, \mathbf{y}^{m}(\tau)\right) d \bar{W}^{i}\right| .
\end{aligned}
$$

We write the stochastic integral as follows

$$
\sum_{i=1}^{m} \int_{0}^{t}\left(g\left(\mathbf{y}^{m}(s), s\right) e_{i}, \mathbf{y}^{m}(s)\right) d \bar{W}^{i}=\sum_{i=1}^{m} \int_{0}^{t} X^{1, i}(s) d \bar{W}^{i}(s),
$$

where

$$
\left[X^{1, i}(s) ; i=1, \ldots m\right]:=\left[\left(g\left(\mathbf{y}^{m}(s), s\right) e_{1}, \mathbf{y}^{m}(s)\right), \ldots,\left(g\left(\mathbf{y}^{m}(s), s\right) e_{m}, \mathbf{y}^{m}(s)\right)\right]
$$

is a row matrix and we view the Brownian motion $\left[\bar{W}^{i} ; i=1, \ldots, m\right]$ as a column vector. The Burkholder-Davis-Gundy's inequality in the form given by [31, Chapter 3, Remark 3.30] enables us to derive the the following estimates

$$
\begin{aligned}
2 \overline{\mathbb{E}} \sup _{s \in\left[0, t \wedge \tau_{M}\right]}\left|\sum_{i=1}^{m} \int_{0}^{s}\left(g\left(\mathbf{y}^{m}(\tau), \tau\right) e_{i}, \mathbf{y}^{m}(\tau)\right) d \bar{W}^{i}\right| & \leq C \overline{\mathbb{E}}\left(\int_{0}^{t \wedge \tau_{M}} \sum_{i=1}^{m}\left(g\left(\mathbf{y}^{m}(s), s\right) e_{i}, \mathbf{y}^{m}(s)\right)^{2} d s\right)^{\frac{1}{2}} \\
& \leq C \overline{\mathbb{E}}\left(\int_{0}^{t \wedge \tau_{M}} \sum_{i=1}^{m}\left|g\left(\mathbf{y}^{m}(s), s\right) e_{i}\right|^{2}\left|\mathbf{y}^{m}(s)\right|^{2} d s\right)^{\frac{1}{2}} \\
& \leq C \overline{\mathbb{E}}\left(\int_{0}^{t \wedge \tau_{M}}|| g\left(\mathbf{y}^{m}(s), s\right)||_{J_{2}}^{2}\left|\mathbf{y}^{m}(s)\right|^{2} d s\right)^{\frac{1}{2}} .
\end{aligned}
$$


From the last line and the assumption on $g$ we deduce thanks to Cauchy's inequality that

$$
\begin{array}{r}
2 \overline{\mathbb{E}} \sup _{s \in\left[0, t \wedge \tau_{M}\right]}\left|\sum_{i=1}^{m} \int_{0}^{s}\left(g\left(\mathbf{y}^{m}(\tau), \tau\right) e_{i}, \mathbf{y}^{m}(\tau)\right) d \bar{W}^{i}\right| \leq C \overline{\mathbb{E}} \int_{0}^{t \wedge \tau_{M}}\left(1+\left|\mathbf{y}^{m}(s)\right|^{2}\right) d s \\
+\frac{1}{2} \overline{\mathbb{E}} \sup _{s \in\left[0, t \wedge \tau_{m}\right]}\left|\mathbf{y}^{m}(s)\right|^{2} .
\end{array}
$$

Now it follows from (53) and (54) that

$$
\overline{\mathbb{E}} \sup _{s \in\left[0, t \wedge \tau_{M}\right]}\left|\mathbf{y}^{m}(s)\right|^{2}+2 \tilde{\nu} \overline{\mathbb{E}} \int_{0}^{t \wedge \tau_{m}} \|\left.\mathbf{y}^{m}(s)\right|_{\mathbb{V}} ^{p, 2} d s \leq\left|\mathbf{y}_{0}\right|^{2}+C \overline{\mathbb{E}} \int_{0}^{t \wedge \tau_{M}}\left(1+\left|\mathbf{y}^{m}(s)\right|^{2}\right) . d s
$$

Since the second term of the left hand side of $(55)$ is positive, then it follow from Gronwall's lemma that

$$
\overline{\mathbb{E}} \sup _{s \in\left[0, t \wedge \tau_{M}\right]}\left|\mathbf{y}^{m}(s)\right|^{2}<C,
$$

for any $t \in\left[0, T_{m}\right]$. Using (56) in (55), we get

$$
\overline{\mathbb{E}} \int_{0}^{t \wedge \tau_{m}}\left\|\mathbf{y}^{m}(s)\right\|_{\mathbb{V}}^{p, 2} d s \leq C .
$$

Since the constants $C$ in (56)-(57) do not depend on $m$ and $M$, then we can show that $\tau_{M} \nearrow T$ $\overline{\mathbb{P}}$-almost surely as $M \rightarrow \infty$. Therefore, $T_{m}=T$. We can conclude by passing to the limit in (56) and (57) that

$$
\overline{\mathbb{E}} \sup _{s \in[0, T]}\left|\mathbf{y}^{m}(s)\right|^{2}<C,
$$

and

$$
\overline{\mathbb{E}} \int_{0}^{T}\left\|\mathbf{y}^{m}(s)\right\|_{\mathbb{V}}^{p, 2} d s \leq C .
$$

Now let $r>2$. Thanks to Itô's formula, we derive from (49) that

$$
\begin{aligned}
\left|\mathbf{y}^{m}(t)\right|^{r}+r \int_{0}^{t}\left|\mathbf{y}^{m}(s)\right|^{r-2}\left\langle\mathcal{A} \mathbf{y}^{m}(s), \mathbf{y}^{m}(s)\right\rangle d s & \leq\left|\mathbf{y}_{0}^{m}\right|^{r}+C \int_{0}^{t}\left|\mathbf{y}^{m}(s)\right|^{r-2}\left[\left\langle f\left(\mathbf{y}^{m}(s), s\right), \mathbf{y}^{m}(s)\right\rangle\right. \\
& \left.+\left\|g\left(\mathbf{y}^{m}(s), s\right)\right\|_{J_{2}}^{2}\right] d s \\
& +r \sum_{i=1}^{m} \int_{0}^{t}\left|\mathbf{y}^{m}(s)\right|^{r-2}\left(g\left(\mathbf{y}^{m}(s), s\right) e_{i}, \mathbf{y}^{m}(s)\right) d \bar{W}^{i} .
\end{aligned}
$$

Owing to (13), (51) and the assumptions on $g$ and $f$ we derive from the last estimate that

$$
\begin{aligned}
\left|\mathbf{y}^{m}(t)\right|^{r}+r \tilde{\nu} \int_{0}^{t}\left|\mathbf{y}^{m}(s)\right|^{r-2}|| \mathbf{y}^{m}(s) \|_{\mathbb{V}}^{p, 2} d s & \leq\left|\mathbf{y}_{0}\right|^{r}+C \int_{0}^{t}\left|\mathbf{y}^{m}(s)\right|^{r-2}\left[C _ { \varepsilon } \left(1+\left|\mathbf{y}^{m}(s)\right|^{2}\right.\right. \\
& \left.\left.+\varepsilon\left\|\mathbf{y}^{m}(s) \mid\right\|_{\mathbb{V}}^{p, 2}+C(\varepsilon, p)\right)+C\left(1+\left|\mathbf{y}^{m}(s)\right|^{2}\right)\right] d s \\
& +r \sum_{i=1}^{m} \int_{0}^{t}\left|\mathbf{y}^{m}(s)\right|^{r-2}\left(g\left(\mathbf{y}^{m}(s), s\right) e_{i}, \mathbf{y}^{m}(s)\right) d \bar{W}^{i} .
\end{aligned}
$$


By an appropriate choice of $\varepsilon$ we deduce from (61) that

$$
\begin{aligned}
\left|\mathbf{y}^{m}(t)\right|^{r}+\tilde{\nu} \int_{0}^{t}\left|\mathbf{y}^{m}(s)\right|^{r-2}|| \mathbf{y}^{m}(s)||_{\mathbb{V}}^{p, 2} d s & \leq\left|\mathbf{y}_{0}\right|^{r}+C \int_{0}^{t}\left|\mathbf{y}^{m}(s)\right|^{r-2}\left(1+\left|\mathbf{y}^{m}(s)\right|^{2}\right) d s \\
& +r \sum_{i=1}^{m} \int_{0}^{t}\left|\mathbf{y}^{m}(s)\right|^{r-2}\left(g\left(\mathbf{y}^{m}(s), s\right) e_{i}, \mathbf{y}^{m}(s)\right) d \bar{W}^{i} .
\end{aligned}
$$

Making use of Young's inequality it is not difficult to prove that there exists $C>0$ such that

$$
\left|\mathbf{y}^{m}(s)\right|^{r-2}\left(1+\left|\mathbf{y}^{m}(s)\right|^{2}\right) \leq C\left(1+\left|\mathbf{y}^{m}(s)\right|^{r}\right) .
$$

Thus, from (62) we see that

$$
\begin{aligned}
\overline{\mathbb{E}} \sup _{s \in[0, t]}\left|\mathbf{y}^{m}(s)\right|^{r}+\tilde{\nu} \overline{\mathbb{E}} \int_{0}^{t}\left|\mathbf{y}^{m}(s)\right|^{r-2}|| \mathbf{y}^{m}(s)||_{\mathbb{V}}^{p, 2} d s \leq C\left(\left|\mathbf{y}_{0}\right|^{r}, T, p\right)+C \overline{\mathbb{E}} \int_{0}^{t}\left|\mathbf{y}^{m}(s)\right|^{r} d s \\
+\left.r \overline{\mathbb{E}} \sup _{s \in[0, t]}\left|\sum_{i=1}^{m} \int_{0}^{s}\right| \mathbf{y}^{m}(\tau)\right|^{r-2}\left(g\left(\mathbf{y}^{m}(\tau), \tau\right) e_{i}, \mathbf{y}^{m}(\tau)\right) d \bar{W}^{i} \mid .
\end{aligned}
$$

By using the Burkholder-Davis-Gundy's inequality as we did in the proof Eq. (54) and the assumptions on $g$ we derive from the last estimate that

$$
\begin{aligned}
\overline{\mathbb{E}} \sup _{s \in[0, t]}\left|\mathbf{y}^{m}(s)\right|^{r}+\tilde{\nu} \overline{\mathbb{E}} \int_{0}^{t}\left|\mathbf{y}^{m}(s)\right|^{r-2} \|\left.\mathbf{y}^{m}(s)\right|_{\mathbb{V}} ^{p, 2} d s & \leq C\left(\left|\mathbf{y}_{0}\right|^{r}, T, p\right)+C \overline{\mathbb{E}} \int_{0}^{t}\left|\mathbf{y}^{m}(s)\right|^{r} d s \\
& +C \overline{\mathbb{E}}\left(\int_{0}^{t}\left|\mathbf{y}^{m}(s)\right|^{2 r-2}\left(1+\left|\mathbf{y}^{m}(s)\right|^{2}\right) d s\right)^{\frac{1}{2}} .
\end{aligned}
$$

As before we can check that the third term of the right hand side of (64) can be bounded from above by

$$
C \overline{\mathbb{E}} \int_{0}^{t}\left|\mathbf{y}^{m}(s)\right|^{r} d s+C
$$

We infer from (64) and (65) that

$$
\overline{\mathbb{E}} \sup _{s \in[0, t]}\left|\mathbf{y}^{m}(s)\right|^{r}+\left.\tilde{\nu} \overline{\mathbb{E}} \int_{0}^{t}\left|\mathbf{y}^{m}(s)\right|^{r-2}|| \mathbf{y}^{m}(s)\right|_{\mathbb{V}} ^{p, 2} d s \leq C\left(\left|\mathbf{y}_{0}\right|^{r}, T, p\right)+C \overline{\mathbb{E}} \int_{0}^{t}\left|\mathbf{y}^{m}(s)\right|^{r} d s .
$$

Making use of Gronwall's inequality in (66), it follows that

$$
\overline{\mathbb{E}} \sup _{s \in[0, t]}\left|\mathbf{y}^{m}(s)\right|^{r}<C, \forall t \in[0, T] \text { and } r>2 .
$$

Next recall that

$$
\left|\mathbf{y}^{m}(t)\right|^{2}+C \int_{0}^{t} \|\left.\mathbf{y}^{m}(s)\right|_{\mathbb{V}} ^{p, 2} d s \leq\left|\mathbf{y}_{0}\right|^{2}+C T+C \int_{0}^{T}\left|\mathbf{y}^{m}(s)\right|^{2} d s+\left|\sum_{i=1}^{m} \int_{0}^{t}\left(g\left(\mathbf{y}^{m}(s) e_{i}, s\right), \mathbf{y}^{m}(s)\right) d \bar{W}^{i}\right| .
$$

Therefore, it is straightforward to check that

$$
\begin{aligned}
\overline{\mathbb{E}}\left(\int_{0}^{T}\left\|\mathbf{y}^{m}(s)\right\|_{\mathbb{V}}^{p, 2} d s\right)^{\frac{r}{2}} & \leq C\left(\left|\mathbf{y}_{0}\right|^{r}, r, T\right)+C \overline{\mathbb{E}}\left(\int_{0}^{T}\left|\mathbf{y}^{m}(s)\right|^{2} d s\right)^{\frac{r}{2}} \\
& +C \overline{\mathbb{E}} \sup _{t \in[0, T]}\left|\sum_{i=1}^{m} \int_{0}^{t}\left(g\left(\mathbf{y}^{m}(s), s\right) e_{i}, \mathbf{y}^{m}(s)\right) d \bar{W}^{i}\right|^{\frac{r}{2}} .
\end{aligned}
$$


Using again the Burkholder-Davis-Gundy's inequality as we did in the proof Eq. (54) and the assumption on $g$ we check that

$$
\begin{array}{r}
\overline{\mathbb{E}} \sup _{t \in[0, T]}\left|\sum_{i=1}^{m} \int_{0}^{t}\left(g\left(\mathbf{y}^{m}(s), s\right) e_{i}, \mathbf{y}^{m}(s)\right) d \bar{W}^{i}\right|^{\frac{r}{2}} \leq C \overline{\mathbb{E}}\left(\int_{0}^{T} \sum_{i=1}^{m}\left(g\left(\mathbf{y}^{m}(s), s\right) e_{i}, \mathbf{y}^{m}(s)\right)^{2} d s\right)^{\frac{r}{4}} \\
\leq C \overline{\mathbb{E}}\left(\int_{0}^{T}\left(1+\left|\mathbf{y}^{m}(s)\right|^{4}\right) d s\right)^{\frac{r}{4}} \\
\leq C T+C T \overline{\mathbb{E}} \sup _{t \in[0, T]}\left|\mathbf{y}^{m}(t)\right|^{r},
\end{array}
$$

where we have used Cauchy-Schwarz's and Cauchy's inequalities. From this last estimate, (67) and (69) we derive that

$$
\overline{\mathbb{E}}\left(\int_{0}^{T}\left\|\mathbf{y}^{m}(s)\right\|_{\mathbb{V}}^{p, 2} d s\right)^{\frac{r}{2}} \leq C, \forall r \in[2, \infty) .
$$

We easily conclude the lemma from (58), (59), (67) and (70).

Now, we derive a crucial estimate on the difference $\mathbf{y}^{m}(t+\theta)-\mathbf{y}^{m}(t)$ in $\mathbb{W}_{s}^{*}$.

Lemma 3.5. Let $s \in \mathbb{R}$ such that $s-1>\frac{n}{2}$. We assume that $t \mapsto \mathbf{y}^{m}(t)$ is extended to zero outside the interval $[0, T]$. Then, there exists a positive constant $C$ such that

$$
\overline{\mathbb{E}} \sup _{|\theta| \in(0, \delta)}\left\|\mathbf{y}^{m}(t+\theta)-\mathbf{y}^{m}(t)\right\|_{\mathbb{W}_{s}^{*}}^{p^{*}} \leq C \delta^{\frac{p^{*}}{p}},
$$

for any $t \in[0, T], m \in \mathbb{N} \backslash\{0\}$ and $\delta \in(0,1)$.

Proof. Noting that $\left\{\left(\sqrt{\lambda_{j}^{1}} \Psi_{j}^{1}, \sqrt{\lambda_{j}^{2}} \Psi_{j}^{2}\right): k=1,2, j=1,2, \ldots\right\}$ forms a basis of $\mathbb{W}_{s}^{*}$, we introduce the projector

$$
P_{m}: \mathbb{W}_{s}^{*} \rightarrow \operatorname{Span}\left\{\left(\sqrt{\lambda_{j}^{1}} \Psi_{j}^{1}, \sqrt{\lambda_{j}^{2}} \Psi_{j}^{2}\right): k=1,2, j=1,2, \ldots, m\right\}
$$

defined by

$$
P_{m} \mathbf{v}=\sum_{j=1}^{m} \lambda_{j}\left\langle\mathbf{v}, \Psi_{j}\right\rangle \Psi_{j}, \quad \forall \mathbf{v} \in \mathbb{W}_{s}^{*} .
$$

The projector $P_{m}$ satisfies (see also [35, Chapitre I, page 76]) the following estimate

$$
\left\|P_{m}\right\|_{\mathcal{L}\left(\mathbb{W}_{s}^{*}, \mathbb{W}_{s}^{*}\right)} \leq 1, \quad \forall m \geq 1 .
$$

In fact, let $\Phi \in \mathbb{W}_{s}$ and $\mathbf{v} \in \mathbb{W}_{s}^{*}$ and $\pi_{m} \Phi:=\sum_{j=1}^{m}\left(\left(\Phi, \Psi_{j}\right)\right)_{s} \Psi_{j}$ be the orthogonal projection from $\mathbb{W}_{s}$ onto $\operatorname{Span}\left\{\left(\Psi_{j}^{1}, \Psi_{j}^{2}\right): k=1,2, j=1,2, \ldots, m\right\} \subset \mathbb{W}_{s}$. By using the definitions of $P_{m}$ and the special basis $\left\{\Psi_{j} ; j=1,2, \ldots\right\}$ we derive that for any $\Phi \in \mathbb{W}_{s}$ and $\mathbf{v} \in \mathbb{W}_{s}^{*}$

$$
\begin{aligned}
\left\langle P_{m} \mathbf{v}, \Phi\right\rangle & =\left\langle\mathbf{v}, \sum_{j=1}^{m} \lambda_{j}\left(\Phi, \Psi_{j}\right) \Psi_{j}\right\rangle \\
=\left\langle\mathbf{v}, \pi_{m} \Phi\right\rangle & \\
& \leq\|\mathbf{v}\|_{\mathbb{W}_{s}^{*}}\left\|\pi_{m} \Phi\right\|_{\mathbb{W}_{s}} \\
& \leq\|\mathbf{v}\|_{\mathbb{W}_{s}^{*}}\|\Phi\|_{\mathbb{W}_{s}},
\end{aligned}
$$

since $\sup _{m \geq 1}\left\|\pi_{m}\right\|_{\mathcal{L}\left(\mathbb{W}_{s}, \mathbb{W}_{s}\right)} \leq 1$. In view of the fact that

$$
\left\|P_{m} \mathbf{v}\right\|_{\mathbb{W}_{s}^{*}}=\sup _{\Phi \in \mathbb{W}_{s}\|\Phi\|_{\mathbb{W}_{s}}=1}\left\langle P_{m} \mathbf{v}, \Phi\right\rangle,
$$


we deduce that

$$
\left\|P_{m} \mathbf{v}\right\|_{\mathbb{W}_{s}^{*}} \leq\|\mathbf{v}\|_{\mathbb{W}_{s}^{*}}
$$

From this last estimate we easily derive the estimate (71).

Now, arguing as in [35, Chapitre I, page 76] we can rewrite the system (42) in the following form

$$
\begin{aligned}
\mathbf{y}^{m}(t) & =\mathbf{y}_{0}^{m}-\int_{0}^{t} P_{m}\left[\mathcal{A} \mathbf{y}^{m}(s)+\mathcal{B}\left(\mathbf{y}^{m}(s), \mathbf{y}^{m}(s)\right)\right] d s+\int_{0}^{t} P_{m} f\left(\mathbf{y}^{m}(s), s\right) d s \\
& +\int_{0}^{t} P_{m} g\left(\mathbf{y}^{m}(s), s\right) e_{i} d \bar{W}^{i},
\end{aligned}
$$

where, for the sake of simplicity, we have set

$$
\int_{0}^{t} P_{m} g\left(\mathbf{y}^{m}(s), s\right) e_{i} d \bar{W}^{i}:=\sum_{i=1}^{m} \int_{0}^{t} P_{m} g\left(\mathbf{y}^{m}(s), s\right) e_{i} d \bar{W}^{i} .
$$

It follows from (72) that

$$
\begin{aligned}
\mathbf{y}^{m}(t+\theta)-\mathbf{y}^{m}(t) & =-\int_{t}^{t+\theta} P_{m}\left[\mathcal{A} \mathbf{y}^{m}(s)+\mathcal{B}\left(\mathbf{y}^{m}(s), \mathbf{y}^{m}(s)\right)\right] d s+\int_{t}^{t+\theta} P_{m} f\left(\mathbf{y}^{m}(s), s\right) d s \\
& +\int_{t}^{t+\theta} P_{m} g\left(\mathbf{y}^{m}(s), s\right) e_{i} d \bar{W}^{i},
\end{aligned}
$$

for any $\theta \in(0, \delta)$ and $\delta \in(0,1)$. From (73) and (71) we infer that

$$
\left\|\mathbf{y}^{m}(t+\theta)-\mathbf{y}^{m}(t)\right\|_{\mathbb{W}_{s}^{*}}^{p^{*}} \leq C I_{1}(t, \theta)+C I_{2}(t, \theta)+C I_{3}(t, \theta)+C I_{4}(t, \theta),
$$

where

$$
\begin{aligned}
& I_{1}(t, \theta)=\left(\int_{t}^{t+\theta}\left\|\mathcal{A} \mathbf{y}^{m}(r)\right\|_{\mathbb{W}_{s}^{*}} d r\right)^{p^{*}}, I_{2}(t, \theta)=\left(\int_{t}^{t+\theta}\left\|\mathcal{B}\left(\mathbf{y}^{m}(r), \mathbf{y}^{m}(r)\right)\right\|_{\mathbb{W}_{s}^{*}} d r\right)^{p^{*}}, \\
& I_{3}(t, \theta)=\left(\int_{t}^{t+\theta}\left\|f\left(\mathbf{y}^{m}(r), r\right)\right\|_{\mathbb{W}_{s}^{*}} d r\right)^{p^{*}}, I_{4}(t, \theta)=\left|\sum_{i=1}^{m} \int_{t}^{t+\theta} g\left(\mathbf{y}^{m}(r), r\right) e_{i} d \bar{W}^{i}\right|^{p^{*}} .
\end{aligned}
$$

For $I_{1}(t, \theta)$, we have

But,

$$
I_{1}(t, \theta) \leq C\left(\int_{t}^{t+\theta}\left\|\mathcal{A} \mathbf{y}^{m}(r)\right\|_{\mathbb{V} *} d r\right)^{p^{*}} .
$$

Therefore

$$
\int_{t}^{t+\theta}\left\|\mathcal{A} \mathbf{y}^{m}(r)\right\|_{\mathbb{V}^{*}} d r \leq \theta^{\frac{1}{p}}\left(\int_{t}^{t+\theta}\left\|\mathcal{A} \mathbf{y}^{m}(r)\right\|_{\mathbb{V}^{*}}^{p^{*}} d r\right)^{p^{*}} .
$$

$$
I_{1}(t, \theta) \leq C \theta^{\frac{p^{*}}{p}} \int_{t}^{t+\theta}\left\|\mathcal{A} \mathbf{y}^{m}(r)\right\|_{\mathbb{V}^{*}}^{p^{*}} d r
$$

and thanks to (14) we have

$$
I_{1}(t, \theta) \leq C \theta^{\frac{p^{*}}{p}} \int_{t}^{t+\theta}\left(1+\left\|\mathbf{y}^{m}(r)\right\|_{\mathbb{V}}^{p, 2}\right) d r
$$

Thus,

$$
\overline{\mathbb{E}} \sup _{\theta \in(0, \delta)} I_{1}(t, \theta) \leq C \delta^{\frac{p^{*}}{p}}\left(\delta+\overline{\mathbb{E}} \int_{0}^{T}\left\|\mathbf{y}^{m}(r)\right\|_{\mathbb{V}}^{p, 2} d r\right) .
$$


Owing to Lemma 3.4 we derive from this last inequality that

$$
\overline{\mathbb{E}} \sup _{\theta \in(0, \delta)} I_{1}(t, \theta) \leq C \delta^{\frac{p^{*}}{p}} .
$$

As above we can check that

$$
I_{2}(t, \theta) \leq C \theta^{\frac{p^{*}}{p}} \int_{t}^{t+\theta}\left\|\mathcal{B}\left(\mathbf{y}^{m}(r), \mathbf{y}^{m}(r)\right)\right\|_{\mathbb{W}_{s}^{*}}^{p^{*}} d r
$$

In view of (34) and Lemma 3.4, we have

$$
\overline{\mathbb{E}} \sup _{\theta \in(0, \delta)} I_{2}(t, \theta) \leq C \delta^{\frac{p^{*}}{p}} .
$$

It follows from the assumptions on $f$ and Lemma 3.4 that

$$
\overline{\mathbb{E}} \sup _{\theta \in(0, \delta)} I_{3}(t, \theta) \leq C \delta^{\frac{p^{*}}{p}} .
$$

Let $\left[X^{1, i}(r) ; i=1, \ldots, m\right]=\left[P_{m} g\left(\mathbf{y}^{m}(r), r\right) e_{1}, \ldots, P_{m} g\left(\mathbf{y}^{m}(r), r\right) e_{m}\right] \in \underbrace{\mathbb{H} \times \ldots \times \mathbb{H}}_{m \text { times }}$ be a row matrix whose entries are $\mathbb{H}$-valued stochastic processes. Define the norm of $\left[X^{1, i}(r) ; i=1, \ldots, m\right]$ by

$$
|X(r)|_{\mathbb{H} \times m}^{2}:=\sum_{i=1}^{m}\left|X^{1, i}(r)\right|^{2} .
$$

We will view the stochastic integral in the definition of $I_{4}$ as follows

$$
\sum_{i=1}^{m} \int_{t}^{t+\theta} g\left(\mathbf{y}^{m}(r), r\right) e_{i} d \bar{W}^{i}:=\sum_{i=1}^{m} \int_{t}^{t+\theta} X^{l, i}(r) d \bar{W}^{i}(r) .
$$

We apply the Burkholder-Davis-Gundy's inequality for Hilbert space-valued processes (see, for instance, [30, Chapter 15, Theorem 15.7]) and derive that

$$
\begin{aligned}
\overline{\mathbb{E}} \sup _{\theta \in(0, \delta)} I_{4}(t, \theta) & \leq C \overline{\mathbb{E}}\left(\int_{t}^{t+\delta} \sum_{i=1}^{m}\left|g\left(\mathbf{y}^{m}(r), r\right) e_{i}\right|^{2} d r\right)^{\frac{p^{*}}{2}}, \\
& \leq C \overline{\mathbb{E}}\left(\int_{t}^{t+\delta}\left\|g\left(\mathbf{y}^{m}(r), r\right)\right\|_{J_{2}}^{2} d r\right)^{\frac{p^{*}}{2}} .
\end{aligned}
$$

By using the assumptions on $g$, we see from the last estimate that

$$
\begin{aligned}
\overline{\mathbb{E}} \sup _{\theta \in(0, \delta)} I_{4}(t, \theta) & \leq C \overline{\mathbb{E}}\left(\int_{t}^{t+\delta}\left(1+\left|\mathbf{y}^{m}(r)\right|^{2}\right) d r\right)^{\frac{p^{*}}{2}} \\
& \leq C\left(\delta+\delta \overline{\mathbb{E}} \sup _{s \in[t, t+\delta]}\left|\mathbf{y}^{m}(r)\right|^{2}\right)^{\frac{p^{*}}{2}} .
\end{aligned}
$$

Thanks to Lemma 3.4 we have that

$$
\overline{\mathbb{E}} \sup _{\theta \in(0, \delta)} I_{4}(t, \theta) \leq C \delta^{\frac{p^{*}}{2}}
$$

Now it follows from (74)-(79) that

$$
\overline{\mathbb{E}} \sup _{\theta \in(0, \delta)}\left\|\mathbf{y}^{m}(t+\theta)-\mathbf{y}^{m}(t)\right\|_{\mathbb{W}_{s}^{*}}^{p^{*}} \leq C \delta^{\frac{p^{*}}{p}},
$$


for any positive integer $m, t \in[0, T]$ and $\delta \in(0,1)$. One can deal with the negative values of $\theta$ using similar arguments. This completes the proof of the lemma.

3.2. Tightness property and passage to the limit. Throughout this subsection we fix $s \in \mathbb{R}$ such that $s-1>\frac{n}{2}$. We set $\mathfrak{S}_{1}=L^{2}(0, T, \mathbb{H}) \cap C\left(0, T ; \mathbb{W}_{s}^{*}\right)\left(\right.$ resp., $\left.\mathfrak{S}_{2}=\mathcal{C}(0, T ; \mathbb{K})\right)$ and we denote by $\mathcal{B}\left(\mathfrak{S}_{1}\right)$ (resp., $\mathcal{B}\left(\mathfrak{S}_{2}\right)$ ) its Borel $\sigma$-algebra. The family of laws of $\left\{\mathbf{y}^{m}: m \in \mathbb{N} \backslash\{0\}\right\}$ is denoted by $\left\{\Pi_{1, m}: m \in \mathbb{N}\right\}$. We will prove the tightness of the family of laws $\left\{\Pi_{1, m}: m \in \mathbb{N}\right\}$, in $\mathfrak{S}_{1}$. For this purpose let us recall a result which will be needed in the sequel; we refer for instance to the book of Métivier [42, Chapter VI, Lemma 2 \& Lemma 3] for its proof.

Lemma 3.6. Let $\mathbb{B}, \mathbb{B}_{0}$ and $\mathbb{B}_{1}$ be three reflexive Banach spaces satisfying the compact embeddings $\mathbb{B}_{0} \hookrightarrow \mathbb{B} \hookrightarrow \mathbb{B}_{1}$. Let $q \in(1, \infty)$ and $\mathcal{K}$ be a subset of $L^{q}(0, T ; \mathbb{B})$ which is included in a compact set of $L^{q}\left(0, T ; \mathbb{B}_{1}\right)$ and

$$
\sup _{\mathbf{u} \in \mathcal{K}} \int_{0}^{T}|\mathbf{u}(s)|_{\mathbb{B}_{0}}^{q} d s<\infty .
$$

Then $\mathcal{K}$ is relatively compact in $L^{q}(0, T ; \mathbb{B})$.

We will apply this lemma on the cartesian product $L^{p, 2}(0, T ; \mathbb{V})$ which is equal to the space of couples $(\mathbf{u} ; \mathbf{v})$ such that $\mathbf{u} \in L^{p}\left(0, T ; \mathbb{V}_{1, p}\right)$ and $\mathbf{v} \in L^{2}\left(0, T ; \mathbb{V}_{2}\right)$. It is a Banach space when endowed with the norm

$$
\|(\mathbf{u} ; \mathbf{v})\|_{L^{p, 2}(0, T ; \mathbb{V})}=\left(\|\mathbf{u}\|_{L^{p}\left(0, T ; \mathbb{V}_{1, p}\right)}^{2}+\|\mathbf{v}\|_{L^{2}\left(0, T ; \mathbb{V}_{2}\right)}^{2}\right)^{\frac{1}{2}} .
$$

Now we state one of the main results of this subsection.

Lemma 3.7. The family $\Pi_{1, m}$ is tight on $\mathfrak{S}_{1}$.

Proof of Lemma 3.7. We first prove the tightness in $C\left(0, T ; \mathbb{W}_{s}^{*}\right)$. Thanks to (46) it is easy to see that for any $R>0$ we have

$$
\overline{\mathbb{P}}\left(\left|\mathbf{y}^{m}(t)\right|_{\mathbb{H}}>R\right) \leq \frac{1}{R^{2}} \overline{\mathbb{E}} \sup _{s \in[0, T]}\left|\mathbf{y}^{m}\right|_{\mathbb{H}}^{2} \leq \frac{C_{2}}{R^{2}},
$$

for any $t \in[0, T]$. Since, by the compact embedding $\mathbb{H} \subset \mathbb{W}_{s}^{*}$, balls in $\mathbb{H}$ are compact for the strong topology in $\mathbb{W}^{*}$, then this implies that the family $\left\{\mathbf{y}^{m}(t): m \in \mathbb{N}\right\}$ is relatively compact in $\mathbb{W}_{s}^{*}$ for any $t \in[0, T]$. Thanks to this fact, Lemma 3.5 and [63, Lemma $\left.1, \mathrm{p} 71\right]$ we derive that the laws of the family $\left\{\mathbf{y}^{m} ; m \in \mathbb{N}\right\}$ are tight in $C\left(0, T ; \mathbb{W}_{s}^{*}\right)$.

The tightness of the laws of the family $\left\{\mathbf{y}^{m}: m \in \mathbb{N}\right\}$ in $C\left(0, T ; \mathbb{W}_{s}^{*}\right)$ means that for any $\varepsilon>0$ there exists a compact subset $K_{\varepsilon}$ of $C\left(0, T ; \mathbb{W}_{s}^{*}\right)$ such that

$$
\overline{\mathbb{P}}\left(\mathbf{y}^{m} \in K_{\varepsilon}\right) \geq 1-\varepsilon, \quad m \in \mathbb{N} .
$$

Note that for any $\varepsilon>0,(47)$ implies that there exists a positive constant $L_{\varepsilon}$ such that

$$
\overline{\mathbb{P}}\left(\int_{0}^{T}\left|\mathbf{y}^{m}(s)\right|_{\mathbb{V}}^{p, 2} d s \leq L_{\varepsilon}\right) \geq 1-\varepsilon, \quad m \in \mathbb{N}
$$

where $\int_{0}^{T}\left|\mathbf{y}^{m}(s)\right|_{\mathbb{V}}^{p, 2} d s=\int_{0}^{T}\left\|\mathbf{y}^{m}(s)\right\|_{\mathbb{V}_{1, p}}^{p}+\int_{0}^{T}\left\|\mathbf{y}^{m}(s)\right\|^{2} d s$. The last inequality is equivalent to

$$
\overline{\mathbb{P}}\left(\left\|\mathbf{y}^{m}(s)\right\|_{L^{p, 2}(0, T ; \mathbb{V})} d s \leq L_{\varepsilon}\right) \geq 1-\varepsilon, \quad m \in \mathbb{N} .
$$

Now let

$$
\mathcal{K}_{\varepsilon}=K_{\varepsilon} \cap\left\{\mathbf{u} \in L^{p, 2}(0, T ; \mathbb{V}):\|\mathbf{u}\|_{L^{p, 2}(0, T ; \mathbb{V})}^{2} \leq L_{\varepsilon}\right\} .
$$

Since $L^{p, 2}(0, T ; \mathbb{V}) \cap C\left(0, T ; \mathbb{W}^{*}\right)$ is continuously embedded in $L^{2}(0, T ; \mathbb{H}) \cap L^{2}\left(0, T ; \mathbb{W}^{*}\right)$, then $\mathcal{K}_{\varepsilon}$ satisfies the conditions of Lemma 3.6. Therefore $\mathcal{K}_{\varepsilon}$ is a relatively compact set of $L^{2}(0, T ; \mathbb{H})$. Moreover $\mathbb{P}\left(\mathbf{y}^{m} \in \mathcal{K}_{\varepsilon}\right) \geq 1-2 \varepsilon, m \in \mathbb{N}$. Thus the tightness of the laws of the family $\left\{\mathbf{y}^{m}: m \in \mathbb{N}\right\}$ in $L^{2}(0, T ; \mathbb{H})$ is established. So the lemma is also proved. 
Before we continue, we should note that it is possible to find a set $\bar{\Omega}^{\prime} \in \overline{\mathcal{F}}$ of measure zero such that $\bar{W}(\bar{\omega}) \in C(0, T ; \mathbb{K})$ for any $\bar{\omega} \in \bar{\Omega} \backslash \bar{\Omega}^{\prime}$. The law of $\bar{W}$ is denoted by $\Pi_{2}$ and $\mathcal{M}(C(0, T ; \mathbb{K}))$ denotes the space of measures on $\left(\mathfrak{S}_{2}, \mathcal{B}\left(\mathfrak{S}_{2}\right)\right)$. For any $m \in \mathbb{N}$, we construct a family of probability laws on $C(0, T ; \mathbb{K})$ by setting

$$
\Pi_{2, m}=\Pi_{2}, \forall m \geq 1 .
$$

Theorem 3.8. Let $\mathfrak{S}=\mathfrak{S}_{1} \times \mathfrak{S}_{2}$ and $\mathcal{B}(\mathfrak{S})$ its Borel $\sigma$-algebra. The family of laws of $\left(\mathbf{y}^{m} ; \bar{W}\right)$ is tight on $\mathfrak{S}$.

Proof. Endowed with the uniform convergence, $C(0, T ; \mathbb{K})$ is a Polish space, then it follows from [5, Theorem 6.8] that $\mathcal{M}(C(0, T ; \mathbb{K}))$ endowed with the Prohorov's metric is a separable and complete metric space. By construction the family of probability laws $\left\{\Pi_{2, m}: m=1,2, \ldots\right\}$ is reduced to one element which is the law of $\bar{W}$ and belongs to $\mathcal{M}(C(0, T ; \mathbb{K}))$. Therefore, invoking [49, Chapter II, Theorem 3.2] we easily deduce that the family $\left\{\Pi_{2, m}: m=1,2, \ldots\right\}$ is tight on $\mathcal{M}(C(0, T ; \mathbb{K}))$. Owing to this fact along with Lemma 3.7 and [33, Corollary 1.3], the family of laws of the joint processes $\left(\mathbf{y}^{m} ; \bar{W}\right)$ is tight on $\mathfrak{S}$.

Proposition 3.9. There exist a Borel probability measure $\mu$ on $\mathfrak{S}$ and a subsequence of $\left(\mathbf{y}^{m}\right)$ such that its laws weakly converge to $\mu$.

Proof. Thanks to the above theorem the laws of $\left(\mathbf{y}^{m}, \bar{W}\right)$ form a tight sequence on $\mathfrak{S}$. Since $\mathfrak{S}$ is a Polish space, we get the result from the application of Prohorov's theorem (see [5, Theorem I.5.1, page 59]).

The following result relates the above convergence in law to almost sure convergence.

Proposition 3.10. There exist a complete probability space $(\Omega, \mathcal{F}, \mathbb{P})$ and a sequence $\left(\overline{\mathbf{y}}^{m}, W_{m}\right)$ of $\mathfrak{S}$-valued random variables defined on $(\Omega, \mathcal{F}, \mathbb{P})$ such that its law is equal to the law of $\left(\mathbf{y}^{m} ; \bar{W}\right)$ on $\mathfrak{S}$. Also, there exists an $\mathfrak{S}$-random variable $(\mathbf{y} ; W)$ defined on $(\Omega, \mathcal{F}, \mathbb{P})$ such that

$$
\begin{aligned}
W_{m} & \rightarrow W \text { in } \mathcal{C}(0, T ; \mathbb{K}) \mathbb{P} \text {-a.s. }, \\
\overline{\mathbf{y}}^{m} & \rightarrow \mathbf{y} \text { in } L^{2}(0, T ; \mathbb{H}) \mathbb{P} \text {-a.s. }, \\
\overline{\mathbf{y}}^{m} & \rightarrow \mathbf{y} \text { in } C\left(0, T ; \mathbb{W}_{s}^{*}\right) \mathbb{P} \text {-a.s.. }
\end{aligned}
$$

Proof. This is just a consequence of Proposition 3.9 and Skorokhod's Theorem in [5, I.6.7, page 70].

Proposition 3.11. Let $Q=\mathcal{I I}^{*}$ where $\mathcal{I}$ is the canonical injection, which is Hilbert-Schmidt, from $\mathbb{H}$ into $\mathbb{K}$. Then the stochastic process $(W(t))_{t \in[0, T]}$ is a $\mathbb{K}$-valued $Q$-Wiener process on $(\Omega, \mathcal{F}, \mathbb{P})$. Furthermore, if $0 \leq s<t \leq T$ then the increments $W(t)-W(s)$ are independent of the $\sigma$-algebra $\mathcal{F}_{s}$ generated by $\mathbf{y}(r), W(r)$ for $r \in[0, s]$.

Proof. We closely follow [9]. By Proposition 3.10 the laws of $\left(\mathbf{y}^{m}, \bar{W}\right)$ are equal to those of $\left(\overline{\mathbf{y}}^{m}, W_{m}\right)$ on $\mathfrak{S}$ and by Remark $2.8, \bar{W}$ is $\mathbb{K}$-valued $Q$-Wiener process. Hence it is easy to check that $W_{m}$ form a sequence of $Q$-Wiener processes taking values in $\mathbb{K}$. Moreover, for $0 \leq s<t \leq T$ the increments $W_{m}(t)-W_{m}(s)$ are independent of the $\sigma$-algebra generated by $\left(\overline{\mathbf{y}}^{m}(r), W_{m}(r)\right)$, for $r \in[0, s]$. Now we will check that $W$ is a $\mathbb{K}$-valued $Q$-Wiener process by showing that its finite dimensional distributions are Gaussian. For this purpose we use characteristic function. Let $k \in \mathbb{N}$ and let $s_{0}=0<s_{1}<\cdots<s_{k} \leq T$. For each $\mathbf{u} \in \mathbb{K},\left(t_{1}, \ldots, t_{k}\right) \in \mathbb{R}^{k}$ we have

$$
\mathbb{E}\left[e^{i \sum_{j=1}^{k} t_{j}\left\langle W_{m}\left(s_{j}\right)-W_{m}\left(s_{j-1}\right), \mathbf{u}\right\rangle_{\mathbb{K}}}\right]=e^{-\frac{1}{2} \sum_{j=1}^{k} t_{j}^{2}\left(s_{j}-s_{j-1}\right)\langle Q \mathbf{u}, \mathbf{u}\rangle_{\mathbb{K}}},
$$


where $i^{2}=-1$. Thanks to (80) and Lebesgue dominated convergence theorem, we have

$$
\begin{aligned}
\lim _{m \rightarrow \infty} \mathbb{E}\left[e^{i \sum_{j=1}^{k} t_{j}\left\langle W_{m}\left(s_{j}\right)-W_{m}\left(s_{j-1}\right), \mathbf{u}\right\rangle_{\mathbb{K}}}\right] & =\mathbb{E}\left[e^{i \sum_{j=1}^{k} t_{j}\left\langle W\left(s_{j}\right)-W\left(s_{j-1}\right), \mathbf{u}\right\rangle_{\mathbb{K}}}\right] \\
& =e^{-\frac{1}{2} \sum_{j=1}^{k} t_{j}^{2}\left(s_{j}-s_{j-1}\right)\langle Q \mathbf{u}, \mathbf{u}\rangle_{\mathbb{K}}}
\end{aligned}
$$

from which we infer that the finite dimensional distributions of $W$ are Gaussian. Next we prove that the increments $W(t)-W(s), 0 \leq s<t \leq T$, are independent of the $\sigma$-algebra generated by $(\mathbf{y}(r), W(r))$ for $r \in[0, s]$. To do so let us consider $\left\{\phi_{j}: j=1, \ldots, k\right\} \subset C_{b}\left(\mathbb{W}_{s}^{*}\right)$ and $\left\{\psi_{j}: j=1, \ldots, k\right\} \subset C_{b}(\mathbb{K})$, where

$$
C_{b}(\mathbf{B})=\{\phi: \mathbf{B} \rightarrow \mathbb{R}, \phi \text { is continuous and bounded }\},
$$

for any Banach space B. Let also $0 \leq r_{1}<\cdots<r_{k} \leq s<t \leq T, \psi \in C_{b}(\mathbb{K})$. For each $m \in \mathbb{N}$, there holds

$$
\begin{aligned}
& \mathbb{E}\left[\left(\prod_{j=1}^{k} \phi_{j}\left(\overline{\mathbf{y}}^{m}\left(r_{j}\right)\right) \prod_{j=1}^{k} \psi_{j}\left(W_{m}\left(r_{j}\right)\right)\right)\right. \\
& \left.\times \psi\left(W_{m}(t)-W_{m}(s)\right)\right] \\
& =\mathbb{E}\left[\prod_{j=1}^{k} \phi_{j}\left(\overline{\mathbf{y}}^{m}\left(r_{i}\right)\right) \prod_{j=1}^{k} \psi_{j}\left(W_{m}\left(r_{j}\right)\right)\right] \\
& \times \mathbb{E}\left(\psi\left(W_{m}(t)-W_{m}(s)\right)\right) .
\end{aligned}
$$

Thanks to (80) and (82) and Lebesgue dominated convergence theorem, the same identity is true with $(\mathbf{y}, W)$ in place of $\left(\overline{\mathbf{y}}^{m}, W_{m}\right)$

Since $\left(\overline{\mathbf{y}}^{m}, W_{m}\right)$ and $\left(\mathbf{y}^{m}, \bar{W}\right)$ have the same law (see Proposition 3.10), it follows from Lemma 3.4 that $\overline{\mathbf{y}}^{m}$ satisfies the estimates

$$
\begin{array}{r}
\mathbb{E} \sup _{t \in[0, T]}\left|\overline{\mathbf{y}}^{m}(t)\right|^{r}<C, \\
\mathbb{E}\left(\int_{0}^{T}\left\|\overline{\mathbf{y}}^{m}(t)\right\|_{\mathbb{V}}^{p, 2} d t\right)^{\frac{r}{2}}<C,
\end{array}
$$

for any $r \in[2, \infty)$. Here $\mathbb{E}$ denotes the mathematical expectation with respect to $\mathbb{P}$. Now arguing exactly as in [9, Proof of (4.12), page 20] we can prove that the stochastic process $\mathbf{y}$ satisfies the following property:

$$
\mathbb{E} \sup _{t \in[0, T]}|\mathbf{y}(t)|^{r}<\infty,
$$

for any $r \in[2, \infty)$. Thanks to Eberlein-Smulyan Theorem (see [72, Chapter 21, Proposition 21.23(h)]) we can extract a subsequence of $\mathbf{y}^{-m}$ denoted by $\mathbf{y}^{-m_{k}}$ such that for any $r \in(2, \infty)$

$$
\begin{aligned}
\overline{\mathbf{y}}^{m_{k}} & \rightarrow \mathbf{y} \text { weakly in } L^{r / 2}\left(\Omega, L^{p, 2}(0, T ; \mathbb{V})\right), \\
\overline{\mathbf{y}}^{m_{k}}(T) & \rightarrow \beta \text { weakly in } L^{2}(\Omega, \mathbb{H}) .
\end{aligned}
$$

We will show in the course of the proof of Proposition 3.18 below that $\beta=\mathbf{y}(T)$ in $L^{2}(\Omega, \mathbb{H})$.

From the estimate (14) in Lemma 2.3 and the estimate (84) we infer that $P_{m_{k}} \mathcal{A} \overline{\mathbf{y}}^{m_{k}}$ is bounded in $L^{2}\left(\Omega, L^{p^{*}, 2}\left(0, T ; \mathbb{V}^{*}\right)\right)$. Therefore one can find $\Gamma \in L^{2}\left(\Omega, L^{p^{*}, 2}\left(0, T ; \mathbb{V}^{*}\right)\right)$ such that

$$
P_{m_{k}} \mathcal{A} \overline{\mathbf{y}}^{m_{k}} \rightarrow \Gamma \text { weakly in } L^{2}\left(\Omega, L^{p^{*}, 2}\left(0, T ; \mathbb{V}^{*}\right)\right),
$$

for any $r \geq 2$. 
Let $r \geq 2$ and let us consider the positive increasing function $\phi(x)=x^{2 r}$, defined on $\mathbb{R}_{+}$. The function $\phi$ obviously satisfies

$$
\lim _{x \rightarrow \infty} \frac{\phi(x)}{x}=\infty
$$

Thanks to the estimates (83) we have

$$
\sup _{m \geq 1} \mathbb{E}\left(\phi\left(\left\|\overline{\mathbf{y}}^{m_{k}}\right\|_{L^{2}(0, T ; \mathbb{H})}^{r}\right)\right)<\infty .
$$

Thanks to uniform integrability criteria in [30, Chapter 3, Exercise 6] we see that $\left\|\overline{\mathbf{y}}^{m_{k}}\right\|_{L^{2}(0, T ; \mathbb{H})}^{r}$ is uniform integrable with respect to the probability measure. Thanks to Vitali's convergence theorem (see, for instance, [30, Chapter 3, Proposition 3.12]) and (81), we obtain that for any $r \in[2, \infty)$

$$
\overline{\mathbf{y}}^{m_{k}} \rightarrow \mathbf{y} \text { strongly in } L^{r}\left(\Omega, L^{2}(0, T ; \mathbb{H})\right) \text {. }
$$

Thus modulo the extraction of a subsequence denoted again with the same symbols we have

$$
\overline{\mathbf{y}}^{m_{k}} \rightarrow \mathbf{y} d \mathbb{P} \otimes d t \text {-a.e. in } \mathbb{H} \text {. }
$$

In view of (90), the continuity of $P_{m_{k}} f$ and the applicability of Vitali's convergence theorem, we derive that

$$
\int_{0} P_{m_{k}} f\left(\overline{\mathbf{y}}^{m_{k}}(s), s\right) d s \rightarrow \int_{0} f(\mathbf{y}(s), s) d s \text { strongly in } L^{2}\left(\Omega, L^{2}\left(0, T ; \mathbb{V}^{*}\right)\right) .
$$

Thanks to (34), (83), and (84) $P_{m_{k}} \mathcal{B}\left(\overline{\mathbf{y}}^{m_{k}}, \overline{\mathbf{y}}^{m_{k}}\right)$ belongs to a bounded set of $L^{2}\left(\Omega, \mathbb{P} ; L^{2}\left(0, T ; \mathbb{W}_{s}^{*}\right)\right)$. Taking advantage of (86) and (89), we will show that

$$
P_{m_{k}} \mathcal{B}\left(\overline{\mathbf{y}}^{m_{k}}, \overline{\mathbf{y}}^{m_{k}}\right) \rightarrow \mathcal{B}(\mathbf{y}, \mathbf{y}) \text { weakly in } L^{2}\left(\Omega, \mathbb{P} ; L^{2}\left(0, T ; \mathbb{W}_{s}^{*}\right)\right)
$$

To this end let

$$
\mathbb{D}=\left\{\Phi=\phi(\omega) \chi(t) \Psi_{j}: \phi(\omega) \in L^{\infty}(\Omega, \mathbb{P}), \chi(t) \in \mathbb{C}_{c}^{\infty}(0, T) \text { and } j=1,2, \ldots\right\},
$$

where $\left\{\Psi_{j} ; j=1,2, \ldots\right\}$ is defined in (41). This set is dense in $L^{2}\left(\Omega, \mathbb{P} ; L^{2}\left(0, T ; \mathbb{W}_{s}\right)\right)$. Owing to [72, Proposition 21.23], the claim (92) is achieved if we prove that

$$
\mathbb{E}\left(\phi(\omega) \int_{0}^{T}\left\langle\mathcal{B}\left(\overline{\mathbf{y}}^{m_{k}}(s), \overline{\mathbf{y}}^{m_{k}}(s)\right)-\mathcal{B}(\mathbf{y}(s), \mathbf{y}(s)), \Psi_{j}\right\rangle \chi(s) d s\right) \rightarrow 0,
$$

for any $\Phi=\phi(\omega) \chi(t) \Psi_{j} \in \mathbb{D}$. For this purpose, we rewrite the last identity in the following form

$$
\mathbb{E}\left(\phi(\omega) \int_{0}^{T}\left\langle\mathcal{B}\left(\overline{\mathbf{y}}^{m_{k}}(s), \overline{\mathbf{y}}^{m_{k}}(s)\right)-\mathcal{B}(\mathbf{y}(s), \mathbf{y}(s)), \Psi_{j}\right\rangle \chi(s) d s\right)=I_{1}+I_{2} .
$$

where

$$
\begin{array}{r}
I_{1}=\mathbb{E}\left(\phi(\omega) \int_{0}^{T}\left\langle\mathcal{B}\left(\overline{\mathbf{y}}^{m_{k}}(s)-\mathbf{y}(s), \overline{\mathbf{y}}^{m_{k}}(s)\right), \Psi_{j}\right\rangle \chi(s) d s\right), \\
I_{2}=\mathbb{E}\left(\phi(\omega) \int_{0}^{T}\left\langle\mathcal{B}\left(\mathbf{y}(s), \mathbf{y}(s)-\overline{\mathbf{y}}^{m_{k}}(s)\right), \Psi_{j}\right\rangle \chi(s) d s\right) .
\end{array}
$$

For fixed $\Phi \in \mathbb{D}$ and $\Xi \in L^{2}\left(\Omega, \mathbb{P} ; L^{2}(0, T ; \mathbb{V})\right)$ the mapping $\Upsilon \mapsto \mathbb{E}\left(\phi(\omega) \int_{0}^{T}\left\langle\mathcal{B}(\Xi, \Upsilon), \Psi_{j}\right\rangle \chi(s) d s\right)$ is a continuous linear functional on $L^{2}\left(\Omega, \mathbb{P} ; L^{2}(0, T ; \mathbb{V})\right)$. Hence by invoking (86) $I_{2}$ converges to 0 as $m \rightarrow \infty$. Next, we easily derive from (34) that

$$
\begin{aligned}
& \left|\mathbb{E}\left(\phi(\omega) \int_{0}\left\langle\mathcal{B}\left(\overline{\mathbf{y}}^{m_{k}}(s)-\mathbf{y}(s), \overline{\mathbf{y}}^{m_{k}}(s)\right), \Psi_{j}\right\rangle \chi(s) d s\right)\right| \leq C\left(\mathbb{E} \int_{0}^{T}\left|\overline{\mathbf{y}}^{m_{k}}(s)-\mathbf{y}(s)\right|\left\|\overline{\mathbf{y}}^{m_{k}}(s)\right\| d s\right) \\
& \times\|\Phi\|_{L^{\infty}(\Omega \times[0, T] \times \mathcal{Q})},
\end{aligned}
$$


which together with $\mathrm{H}^{*}$ older's inequality and (84) imply that

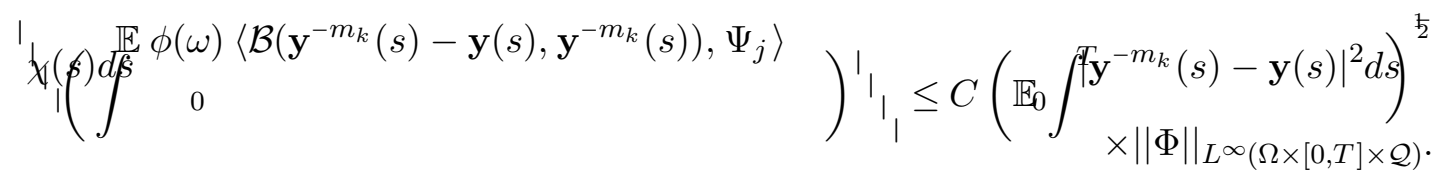

Thanks to (89) the left hand side of this last inequality will converge to 0 as $m \rightarrow \infty$. Hence we have proved that $I_{1}$ converges to 0 as $m \rightarrow \infty$ which also shows that (92) holds.

Now for any $t \in[0, T]$ let

$$
\begin{aligned}
& \mathfrak{M}^{m_{k}}(t)=\mathbf{y}^{-m_{k}}(t)-\mathbf{y}_{0}^{-m_{k}} \int_{0}^{t}\left(P_{m_{k}} \mathcal{A} \mathbf{y}^{-m_{k}}(s)+P_{m_{k}} \mathcal{B}\left(\mathbf{y}^{-m_{k}}(s), \mathbf{y}^{-m_{k}}(s)\right)\right) \int_{0}^{t} P_{m_{k}} f\left(\mathbf{y}^{-m_{k}}(s), s\right) d s \\
& + \text { and } \\
& \left.\mathcal{M}(t)=\mathbf{y}(t)-\mathbf{y}_{0}+\underset{0}{[\Gamma(s)}+\mathcal{B}\left(\mathbf{y}(s), \mathbf{y} \int s\right) \hat{f}\right]^{t} d s-{ }_{0} f(\mathbf{y}(s), s) d s .
\end{aligned}
$$

With the convergence (85), (86), (88),(91), and (92) we see that

$$
\mathfrak{M}^{m_{k}} \rightarrow \mathcal{M} \text { weakly in } L^{2}\left(\Omega, L^{p^{*}, 2}\left(0, T, \mathbb{V}^{*}\right)\right) .
$$

For any $t \geq 0$ and $k \in \mathbb{N}$, let

$$
\begin{array}{r}
\tilde{\mathcal{F}_{t}}:=\sigma\left(\left(\mathbf{y}^{-m_{k}}(s), W_{m_{k}}(s)\right) ; s \leq t, k \in \mathbb{N}\right), \\
\tilde{\mathcal{F}_{t}}:=\sigma((\mathbf{y}(s), W(s)) ; s \leq t),
\end{array}
$$

be the natural filtration generated by $\left(\mathbf{y}^{-m_{k}}, W_{m_{k}}\right)$ and $(\mathbf{y}, W)$, respecty. Let $\mathcal{N}$ be the set of null sets $\mathcal{F}$. For any $t \geq 0$ and $k \in \mathbb{N}$, we denote by $\mathcal{F}_{t}^{m_{k}}:=\bigcap_{s>t} \sigma \quad\left(\tilde{f}_{s} m_{k} \vee \mathcal{F} \bigcup_{\mathcal{J}} \mathcal{Y}\right.$ the join of $\tilde{\mathcal{F}}^{m_{k}}$ and $\tilde{\mathcal{F}_{t}}$, i.e. the coarsest $\sigma$-algebra containing both of $\tilde{\mathcal{F}}^{\sim} m_{t}$ and $\tilde{\mathcal{F}}_{t}$. We endow the new probability system $(\Omega, \mathcal{F}, \mathbb{P})$ with the filtration $\mathbb{F}^{m_{k}}=\left\{\mathcal{F}_{t}^{m_{k}} ; t \geq 0\right\}$. Note that the $\mathbb{W}_{s}{ }^{*}$-valued stochastic processes $\mathbf{y}^{-m_{k}}$ and $\mathbf{y}$ are adapted wrt this filtration. Thus, they are also predictable in $\mathbb{W}_{s}^{*}$ because their sample paths are continuous in $\mathbb{W}_{s}^{*}$.

Remark 3.12. (i) Let $s>1-{ }^{n}{ }_{2}$. Since $g: \mathbb{H} \times[0, T] \rightarrow J_{2}(\mathbb{H})$ is continuous and both $\mathbb{H} \times[0, T]$ and $J_{2}(\mathbb{H})$ are complete, then $g$ is also Cauchy-continuous (i.e. it maps any Cauchy sequence of $\mathbb{H} \times[0, T]$ to a Cauchy sequence of $\left.J_{2}(\mathbb{H})\right)$. Hence, by the density of $\mathbb{H} \times[0, T]$ in $\mathbb{W}_{s}{ }^{*} \times[0, T]$ we can apply $[66$, Theorem 5.5 .3$]$ to justify the existence of a continuous map $g^{\sim}: \mathbb{W}_{s}{ }^{*} \times[0, T] \rightarrow J_{2}(\mathbb{H})$ which extends $g$ and satisfies Assumption $(\mathrm{G})$ on $\mathbb{W}_{s}{ }^{*} \times[0, T]$. Thanks to this remark and the predictability of $\mathbf{y}^{-m_{k}}$ and $\mathbf{y}$, we can define the stochastic integrals

$$
\left.\tilde{\mathcal{M}}_{m_{k}}(t):=\int_{0}^{t} P_{m_{k}} g^{\sim}\left(\mathbf{y}^{-m_{k}}(r), r\right) d W_{m_{k}}(r), \quad \sim(t)\right):=\int_{0}^{t} g^{\sim}(\mathbf{y}(r), r) d W(r), t \in[0, T] .
$$

(ii) Next we note that from (82) and (85), it follows that almost surely $\mathbf{y} \in C\left(0, T ; \mathbb{W}_{s}^{*}\right) \cap$ $L^{\infty}(0, T ; \mathbb{H})$. Hence we deduce from $[68$, Theorem 2.1$]$ that $\mathbb{P}$-a.s. $\mathbf{y} \in C\left(0, T ; \mathbb{H}_{w}\right)$ where $C\left(0, T, \mathbb{H}_{w}\right)$ denotes the space of weakly continuous functions $u:[0, T] \rightarrow \mathbb{H}$. Investigating closely the proof of [68, Theorem 2.1], we infer from [68, Eq. (2.1), page 544] that $\mathbb{P}$-a.s. $\mathbf{y}(t) \in \mathbb{H}$ for all $t \in[0, T]$. The same argument is used to prove that $\mathbb{P}$-a.s. $\mathbf{y}^{-m_{k}}(t) \in \mathbb{H}$ for all $t$ $\in[0, T]$. Since almost surely $\mathbf{y}(t) \in \mathbb{H}$ and $\mathbf{y}^{-m_{k}}(t)$ for all $t \in[0, T]$ and $g^{\sim}$ is an extension of $g:[0, T] \times \mathbb{H} \rightarrow J_{2}(\mathbb{H})$ then w e can identify resp ectiv ely the processes $\mathcal{M}_{k}$ and $\tilde{\mathcal{M}}$ defined above with $f_{0} P_{m_{k}} g\left(\mathbf{y}^{m}{ }^{k}(r), r\right) d W_{m_{k}}(r)$ and $\iint_{0} g(\mathbf{y}(r), r) d W(r), t \in[0, T]$. Hereafter, we will make such identification.

The next proposition is very crucial for our purpose. 
Proposition 3.13. The following identity holds $\mathbb{P}$-a.s.

for any $t \in[0, T]$.

$$
\mathcal{M}(t)=\int_{0}^{t} g(\mathbf{y}(s), s) d W(s)
$$

To prove this proposition we will need the next two lemmata.

Lemma 3.14. We have the following convergence

$$
\mathcal{M}_{m_{k}}^{1}(\cdot):=\int_{0} P_{m_{k}} g\left(\mathbf{y}^{-m_{k}}(r), r\right) d W_{m_{k}} \rightarrow \int_{0} g(\mathbf{y}(r), r) d W
$$

in $L^{2}\left(\Omega, L^{2}(0, T ; \mathbb{H})\right)$.

Proof. We will show that $\mathcal{M}^{1}{ }_{m_{k}}(t)$ converges in $L^{2}(\Omega ; \mathbb{H})$ to $\int^{t} g(\mathbf{y}(r), r) d W(r)$ as $m_{k} \rightarrow \infty$. For this aim let $\varepsilon>0$ arbitrary and let $N \in \mathbb{N}$ such that the partition $\left\{r_{j}^{N}=\underset{N}{j} ; j=0,1, \ldots, N\right\}$ of $[0, T]$ satisfies $\left|r_{j}^{N}-r_{j}^{N}\right|<\varepsilon / 2$ for any $j=0,1, \ldots, N$.

Let $s>1-\frac{n}{2}$ and $g^{\sim}: \mathbb{W}_{s}{ }^{*} \times[0, T] \rightarrow J_{2}(\mathbb{H})$ be a continuous extension of $g$ satisfying Assumption

$(\mathrm{G})$ on $\mathbb{W}_{s}^{*} \times[0, T]$. The justification of the existence of such extension was already done in

Remark 3.12-(i). Thanks to the continuity of $P_{m_{k}} g^{\sim}$ and (82), we can argue as in the proof of (90) to derive that

$$
P_{m_{k}} g^{\sim}\left(\mathbf{y}^{-m_{k}}(r), r\right) \rightarrow g^{\sim}(\mathbf{y}(r), r) \text { strongly in } J_{2}(\mathbb{H}) d \mathbb{P} \otimes d t \text { - a.e.. (95) Since, by }
$$

(95), (83) and Assumption (G), Vitali's convergence theorem is applicable, we derive that

$$
P_{m_{k}} g^{\sim}\left(\mathbf{y}^{-m_{k}}(r), r\right) \rightarrow g^{\sim}(\mathbf{y}(r), r) \text { strongly in } L^{4}\left(\Omega, L^{4}\left(0, T, J_{2}(\mathbb{H})\right)\right) .
$$

Let

$$
R_{1}=\mathbb{E}\left\|\int_{0}^{\|}\right\| P_{m_{k}} g^{\sim}\left(\mathbf{y}^{-m_{k}}(r), r\right) \underset{j=\sigma}{-} \sum_{m_{k}}^{-1} g^{\sim}\left(\mathbf{y}^{-m_{k}}\left(r_{j}^{N}\right), \mathbb{1}_{\left(r_{j}^{N}, r_{j+1}^{N}\right]}(r)\right] d W_{m_{k}}(r){ }_{\mathbb{H}^{N}}^{\|\|^{2} \|} \|
$$

Since $\mathbf{y}^{-m_{k}} \in L^{2}\left(\Omega, C\left(0, T ; \mathbb{W}_{s}^{*}\right)\right)$ and $g^{\sim}$ satisfies Assumption $(\mathrm{G})$ on $\mathbb{W}_{s}{ }^{*} \times[0, T]$, it follows that the map $P_{m_{k}} g^{\sim}\left(\mathbf{y}^{-} m_{k}(\cdot), \cdot\right) \in L^{2}\left(\Omega, C\left(0, T ; J_{2}(\mathbb{H})\right)\right)$. Hence $P_{m_{k}} g^{\sim}\left(\mathbf{y}^{-} m_{k}(\cdot), \cdot\right) \in C\left(0, T ; L^{2}(\Omega\right.$;

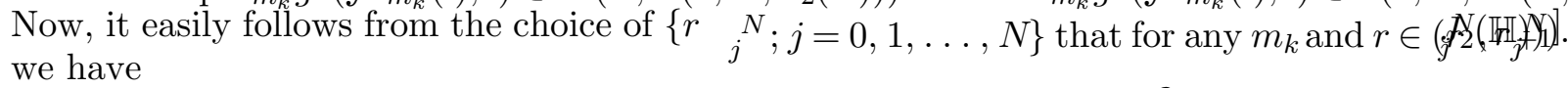

$$
\left.\mathbb{E} \| P_{m_{k}} g^{\sim}\left(\mathbf{y}^{-m_{k}}(r), r\right)-P_{m_{k}} g^{\sim}\left(\mathbf{y}_{j}^{-m_{N k}}\right), r_{r_{j}}^{N}\right) \|_{J_{2}}^{2} \frac{\varepsilon}{4 c T} .
$$

We also have the following chain of inequalities

$$
\begin{aligned}
& R_{1} \leq c \mathbb{E} \int_{0}^{T} \| P_{m_{k}} g^{\sim}\left(\mathbf{y}^{-m_{k}}(r), r\right) \stackrel{N}{-} \sum_{j=\sigma} P_{m_{k}}^{-1} g^{\sim}\left(\mathbf{y}^{-m_{k}}\left(r_{j}{ }_{r_{j}}^{N}\right), \mathbb{1}_{\left(r_{j}^{N}, r_{j+1}^{N}\right]}(r) \|_{J_{2}}^{2} d r\right.
\end{aligned}
$$

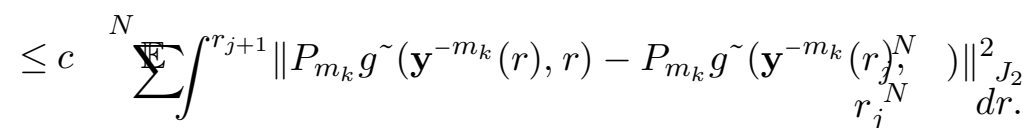

From the last line and (97) we infer that for any $\varepsilon>0$ there exists $k_{0}$ such that for $m_{k}>k_{0}$ we have

Set

$$
R_{1} \leq \frac{\varepsilon}{4}
$$

$$
R_{4}=\mathbb{E} \int_{0}^{\|}\|\|_{0}^{t}\left(P_{m_{k}} g^{\sim}(\mathbf{y}(r), r)-g^{\sim}(\mathbf{y}(r), r)\right) d W \stackrel{\|^{2}}{\left(r^{N}\right)}\left\|^{H}\right\|
$$


Using the It ^o's isometry for stochastic integral in the righthand side of the above estimate we derive that

$$
R_{4} \leq C \mathbb{E}\left\|\int_{0}^{T} m_{k} g^{\sim}(\mathbf{y}(r), r)-g^{\sim}(\mathbf{y}(r), r)\right\|_{J_{2}}^{2} d r
$$

from which and (96) we infer that for any $\varepsilon>0$ there exists $k_{1}$ such that for $m_{k}>k_{1}$ we have

$$
R_{4} \leq \frac{\varepsilon}{4} .
$$

Define

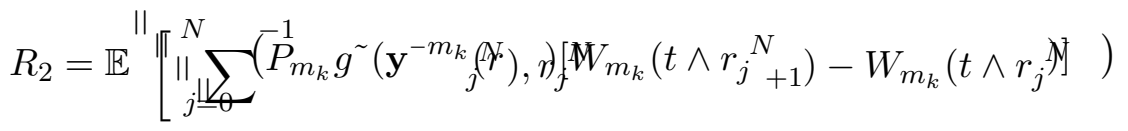

$$
\begin{aligned}
& -\sum_{j=\sigma}^{N}\left(\stackrel{P}{P}_{m_{k}} g^{\sim}\left(\mathbf{y}\left(\hat{b}_{j} \hat{r}_{j}^{N}\right)\left[W\left(t \wedge r_{j+1}^{N}\right)-W\left(t \wedge r_{j}\right){ }^{N}\right)\right]_{\|\mathbb{H}\|_{\|}}{ }^{2}\right.
\end{aligned}
$$

By using the Cauchy-Schwarz's inequality, it is easy to see that

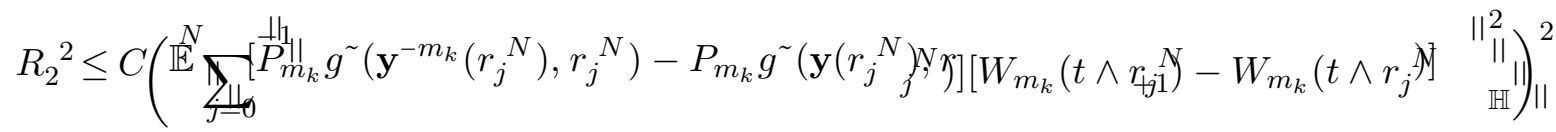

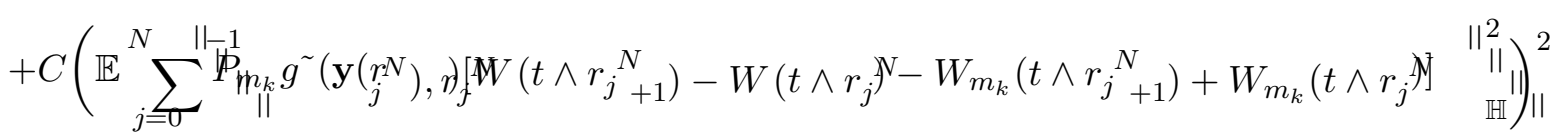

$$
\begin{aligned}
& \left.\leq C \sum_{j=\sigma}^{N} \stackrel{-1}{\mathbb{E}}\left\|P_{m_{k}} g^{\sim}\left(\mathbf{y}^{-m_{k}}\right)\left(, r_{j j}^{N}\right)-P_{m_{k}} g^{\sim}\left(\mathbf{y}\left(r_{j}\right)^{N}, r_{j}^{N}\right)\right\|_{J_{2}}^{4} \mathbb{E} \| W_{m_{k}}\left(t \wedge r_{j}{ }^{N}{ }^{\prime}\right)-W_{m_{k}}\left(t \wedge f^{N}\right)\right] \|_{\mathbb{K}}^{4}
\end{aligned}
$$

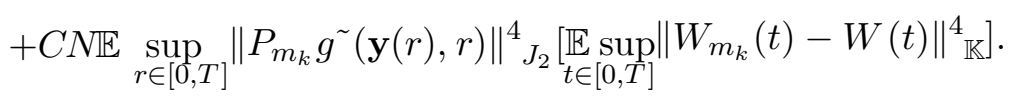

Since $W_{m_{k}}$ is a $Q$-Wiener process with values in $\mathbb{K}$ there exists $C>0$ such that

$$
\mathbb{E}\left\|W_{m_{k}}(r)-W_{m_{k}}(s)\right\|_{\mathbb{K}}^{4} \leq C(r-s)^{2},
$$

for any $0 \leq s<r \leq T$. Hence

$$
\begin{aligned}
& R_{2}^{2} \leq C_{N}^{\underline{T}} \mathbb{E}_{j=0}^{N} \|^{-1} P_{m_{k}} g\left(\mathbf{y}^{-m_{k}}\left(r_{j}^{N}\right), \eta_{j}{ }^{N} P_{m_{k}} g^{\sim}\left(\mathbf{y}\left(r_{j}\right)^{N}, r_{j}^{N}\right) \|_{J_{2}}^{4}\left(r_{j}{ }^{N}+1-r_{j}{ }^{N}\right)\right. \\
& +C N \mathbb{E} \sup _{r \in[0, T]} \| P_{m_{k}} g^{\sim}\left(\mathbf{y}\left(r_{j}^{N}\right), \gamma\right) f_{j}^{\mathbb{N}}\left[\mathbb{E} \sup _{t \in[0, T]}\left\|W_{m_{k}}(t)-W(t)\right\|_{\mathbb{K}}^{4}\right] \\
& \leq C_{N}^{\underline{T}} \mathbb{E} \int_{0}^{T} \|\left[P_{m_{k}} g\left(\mathbf{y}^{-m_{k}}(s), s\right)-P_{m_{k}} g^{\sim}(\mathbf{y}(s), s) \|_{J_{2}}^{4} d s\right. \\
& +C N \mathbb{E} \sup _{r \in[0, T]}\left\|P_{t \in\left[0, T_{i}\right]} g^{\sim}(\mathbf{y}(r), r)\right\|_{J_{2}}^{4}\left[\mathbb{E} \sup \left\|W_{m_{k}}(t)-W(t)\right\|_{\mathbb{K}}^{4}\right] .
\end{aligned}
$$

Thanks to (85) and the fact that $g^{\sim}$ also satisfies Assumption (G), we have

$$
\mathbb{E} \sup _{r \in[0, T]}\left\|P_{m_{k}} g^{\sim}(\mathbf{y}(r), r)\right\|_{J_{2}}^{4}<\infty .
$$

Since $W_{m_{k}}$ is a $Q$-Wiener process with values in $\mathbb{K}$, then for any $q \geq 1$ there exists $C>0$ such that

$$
\mathbb{E} \sup _{t \in[0, T]}\left\|W_{m_{k}}(s)\right\|_{\mathbb{K}}^{q} \leq C T^{\underline{q}} .
$$


Therefore from the almost sure convergence (80), the applicability of Vitali's theorem and (96) we derive that for any $\varepsilon>0$ there exists $k_{2} \in \mathbb{N}$ such that if $m_{k}>k_{2}$ we have

$$
R_{2}^{2} \leq \frac{\varepsilon^{2}}{16}
$$

Let

$$
R_{3}=\mathbb{E}\left\|\int_{0}^{t}\left(P_{m_{k}} \tilde{g}(\mathbf{y}(r), r)-\sum_{j=0}^{N-1}\left[P_{m_{k}} \tilde{g}\left(\mathbf{y}\left(r_{j}^{N}\right), r_{j}^{N}\right) \mathbb{1}_{\left(r_{j}^{N}, r_{j+1}^{N}\right]}(r)\right]\right) d W(r)\right\|_{\mathbb{H}}^{2} .
$$

Arguing again as in the case for $R_{4}$ we get that

$$
R_{3} \leq C \mathbb{E} \int_{0}^{t}\left\|\tilde{g}(\mathbf{y}(r), r)-\sum_{j=0}^{N-1}\left[\tilde{g}\left(\mathbf{y}\left(r_{j}^{N}\right), r_{j}^{N}\right) \mathbb{1}_{\left(r_{j}^{N}, r_{j+1}^{N}\right]}(r)\right]\right\|_{J_{2}}^{2} d s,
$$

from which along the same argument as in $R_{1}$ we easily infer that for any $\varepsilon>0$ there exists $k_{3} \in \mathbb{N}$ such that if $m_{k}>k_{3}$ we have

$$
R_{3} \leq \frac{\varepsilon}{4}
$$

Noticing that

$$
\mathbb{E}\left\|\int_{0}^{t} P_{m_{k}} \tilde{g}\left(\overline{\mathbf{y}}^{m_{k}}(r), r\right) d W_{m_{k}}(r)-\int_{0}^{t} \tilde{g}(\mathbf{y}(r), r) d W(r)\right\|_{\mathbb{H}}^{2} \leq c \sum_{j=1}^{4} R_{j}
$$

We derive from (98), (99), (100) and (101) that for any $\varepsilon>0$ there exists $\bar{k}_{0} \in \mathbb{N}$ (for e.g. $\left.\bar{k}_{0}=\max \left\{k_{0}, k_{1}, k_{2}, k_{3}\right\}\right)$ such that if $m_{k}>\bar{k}_{0}$ we have

$$
\mathbb{E}\left\|\int_{0}^{t} P_{m_{k}} \tilde{g}\left(\overline{\mathbf{y}}^{m_{k}}(r), r\right) d W_{m_{k}}(r)-\int_{0}^{t} \tilde{g}(\mathbf{y}(r), r) d W(r)\right\|_{\mathbb{H}}^{2} \leq \varepsilon .
$$

Since, by Remark 3.12-(ii), we have $\mathbb{P}$-a.s. $\mathbf{y}(t) \in \mathbb{H}$ and $\overline{\mathbf{y}}^{m_{k}}(t)$ for all $t \in[0, T]$ and $\tilde{g}$ is an extension of $g:[0, T] \times \mathbb{H} \rightarrow J_{2}(\mathbb{H})$ then we easily infer that for any $\varepsilon>0$ there exists $\bar{k}_{0} \in \mathbb{N}$ such that if $m_{k}>\bar{k}_{0}$ we have

$$
\mathbb{E}\left\|\int_{0}^{t} P_{m_{k}} g\left(\overline{\mathbf{y}}^{m_{k}}(r), r\right) d W_{m_{k}}(r)-\int_{0}^{t} g(\mathbf{y}(r), r) d W(r)\right\|_{\mathbb{H}}^{2} \leq \varepsilon,
$$

from which we conclude the proof.

Remark 3.15. Let $w$ be real-valued Brownian motion defined on a filtered complete probability space $(\Omega, \mathcal{F}, \mathbb{F}, \mathbb{P})$ and $\phi$ be a predictable process such that $\phi \in L^{2}(\Omega \times[0, T] ; \mathbb{R})$. It is clear that we can consider the stochastic integral $\int_{0}^{*} \phi(s) d w(s)$ as a function $I(\phi, w)$ of the integrand $\phi$ and the Brownian motion $w$. One can prove by making use of the BDG inequality that $I(\cdot, w): L^{2}(\Omega \times[0, T] ; \mathbb{R}) \ni \phi \mapsto \int_{0}^{*} \phi(s) d w(s) \in L^{2}(\Omega \times[0, T] ; \mathbb{R})$ is continuous. However, we do not know whether $I(\phi, \cdot)$ have some continuity property wrt the variable $w$. And this is the reason why we used the discretization method in the proof of Lemma 3.14.

Next we show that $\overline{\mathbf{y}}^{m_{k}}$ is the solution of a finite dimensional SDEs on the new probability system $(\Omega, \mathcal{F}, \mathbb{P})$ which is equipped with the filtration $\mathbb{F}^{m_{k}}$ that was defined above.

Lemma 3.16. The following holds $\mathbb{P}$-a.s

$$
\begin{aligned}
\overline{\mathbf{y}}^{m_{k}}(t)+\int_{0}^{t} P_{m_{k}} \mathcal{A} \overline{\mathbf{y}}^{m_{k}}(s)+P_{m_{k}} \mathcal{B}\left(\overline{\mathbf{y}}^{m_{k}}(s), \overline{\mathbf{y}}^{m_{k}}(s)\right) d s= & \overline{\mathbf{y}}_{0}^{m_{k}}+\int_{0}^{t} P_{m_{k}} f\left(\overline{\mathbf{y}}^{m_{k}}(s), s\right) d s \\
& +\int_{0}^{t} P_{m_{k}} g\left(\overline{\mathbf{y}}^{m_{k}}(s), s\right) d W_{m_{k}},
\end{aligned}
$$

for any $t \in[0, T]$. 
Proof. For any $t \in[0, T]$ let $I^{m_{k}}(t)=\int_{0}^{t} P_{m_{k}} g\left(\overline{\mathbf{y}}^{m_{k}}(s), s\right) d W_{m_{k}}(s)$ and

$$
\begin{array}{r}
\mathcal{M}^{m_{k}}(t)=\mathbf{y}^{m_{k}}(t)+\int_{0}^{t} P_{m_{k}} \mathcal{A} \mathbf{y}^{m_{k}}(s)+P_{m_{k}} \mathcal{B}\left(\mathbf{y}^{m_{k}}(s), \mathbf{y}^{m_{k}}(s)\right) d s-\mathbf{y}_{0}^{m_{k}}-\int_{0}^{t} P_{m_{k}} f\left(\mathbf{y}^{m_{k}}(s), s\right) d s \\
-\sum_{i=1}^{m} \int_{0}^{t} P_{m_{k}} g\left(\mathbf{y}^{m_{k}}(s), s\right) e_{i} d \bar{W}^{i} .
\end{array}
$$

First notice that the stochastic processes $\mathfrak{M}^{m_{k}}$ and $I^{m_{k}}$ are finite dimensional. Next, since the laws of $\left(\mathbf{y}^{m_{k}}, \bar{W}\right)$ and $\left(\overline{\mathbf{y}}^{m_{k}}, W_{m_{k}}\right)$ are equal on $\mathfrak{S}$ and $\overline{\mathbb{P}}$-a.s. $\mathcal{M}^{m_{k}}(t)=0$ for all $t \in[0, T]$, it follows from [64, Lemma 139] that $\mathbb{P}$-a.s. $\mathfrak{M}^{m_{k}}(t)=I^{m_{k}}(t)$ for any $t \in[0, T]$.

Now we prove Proposition 3.13.

Proof of Proposition 3.13. For any $t \in[0, T]$ let $I(t)=\int_{0}^{t} g(\mathbf{y}(s), s) d W$. It follows from (94) that $I^{m_{k}} \rightarrow I$ strongly in $L^{2}\left(\Omega ; L^{2}(0, T, \mathbb{H})\right)$, and from Lemma 3.13 we derive that $\mathfrak{M}^{m_{k}}=I^{m_{k}}$ in $L^{2}\left(\Omega, L^{p^{*}}\left(0, T ; \mathbb{W}_{s}^{*}\right)\right)$ with $s-1>\frac{n}{2}$. Hence we derive from (93) and the uniqueness of the weak limit that $\mathcal{M}=I$ in $L^{2}\left(\Omega, L^{p^{*}}\left(0, T ; \mathbb{W}_{s}^{*}\right)\right)$. This fact implies that for almost all $t \in[0, T]$ and $\mathbb{P}$-a.s. $\mathcal{M}(t)=I(t)$. Since $\mathcal{M}(t)$ and $I(t)$ are $\mathbb{W}_{s}^{*}$-valued continuous functions which agree for almost all $t \in[0, T]$, they must be equal for all $t \in[0, T]$. This ends the proof of the proposition.

The stochatsic process $\mathbf{y}$ satisfies the property stated in the following proposition.

Proposition 3.17. For any $r \in[2, \infty)$ we have $\mathbf{y} \in L^{r}(\Omega, C(0, T ; \mathbb{H}))$.

Proof. Thanks to Proposition 3.13 we have

where

$$
\mathbf{y}(t)=\mathbf{y}_{0}+\int_{0}^{t} G(s) d s+\int_{0}^{t} S(s) W(s), t \in[0, T],
$$

$$
G(\cdot):=\Gamma(\cdot)+\mathcal{B}(\mathbf{y}(\cdot), \mathbf{y}(\cdot))+f(\mathbf{y}(\cdot), \cdot)
$$

and $S(\cdot):=g(\mathbf{y}(\cdot), \cdot)$. Thanks to (88), (92) and (91) we have $\left.G(\cdot) \in L^{2}\left(\Omega, L^{p^{*}, 2}(0, T] ; \mathbb{W}_{s}^{*}\right)\right)$. Owing to (85) and Assumption $(\mathrm{G})$ we obtain $S(\cdot) \in L^{2}\left(\Omega \times[0, T] ; J_{2}(\mathbb{H})\right)$. Now it easily follows from [29, Chapter I, Theorem 3.2] that there exists $\Omega^{*} \in \mathbb{F}$ such that $\mathbb{P}\left(\Omega^{*}\right)=1$ and for each $\omega \in \Omega^{*}$ the function $\mathbf{y}(\cdot)$ takes values in $\mathbb{H}$, and it is continuous in $\mathbb{H}$ with respect to $t$. Now it follows from the item $(85)$ that $\mathbf{y} \in L^{r}(\Omega, C(0, T ; \mathbb{H}))$.

To complete the proof of Theorem 3.3 we need two additional results that we state as propositions.

Proposition 3.18. We have the following identity

$$
\Gamma=\mathcal{A} \mathbf{y} \text { in } L^{2}\left(\Omega, L^{p^{*}, 2}\left(0, T, \mathbb{V}^{*}\right)\right) .
$$

Before we proceed to the proof of this proposition we state and prove the following lemma.

Lemma 3.19. Let $p \geq 2$ and $\mathbf{y}:=(\mathbf{u} ; \mathbf{B}) \in L^{r}(\Omega, C(0, T ; \mathbb{H})) \cap L^{r / 2}\left(\Omega, L^{p, 2}(0, T ; \mathbb{V})\right)$ for any $r \geq 2$. For any $\mathbf{v} \in L^{p}\left(\Omega \times[0, T] ; \mathbb{V}_{1, p}\right)$ and $\mathbf{C} \in L^{2}\left(\Omega \times[0, T] ; \mathbb{V}_{2}\right)$ set

$$
\begin{aligned}
\mathcal{B}_{1}(\mathbf{y}, \mathbf{y}, \mathbf{v}) & =b(\mathbf{u}, \mathbf{u}, \mathbf{v})-\mu b(\mathbf{B}, \mathbf{B}, \mathbf{v}), \\
\mathcal{B}_{2}(\mathbf{y}, \mathbf{y}, \mathbf{C}) & :=\mu b(\mathbf{u}, \mathbf{B}, \mathbf{C})-\mu b(\mathbf{B}, \mathbf{u}, \mathbf{C}) .
\end{aligned}
$$

Then, for any $\mathbf{v} \in L^{p}\left(\Omega \times[0, T] ; \mathbb{V}_{1, p}\right)$ and $\mathbf{C} \in L^{2}\left(\Omega \times[0, T] ; \mathbb{V}_{2}\right)$

(i) $\mathcal{B}_{1}(\mathbf{y}, \mathbf{y}, \mathbf{v}) \in L^{1}(\Omega \times[0, T])$ provided that $p \geq \max \left(1+\frac{2 n}{n+2}, \frac{n+2}{4}\right)$,

(ii) $\mathcal{B}_{2}(\mathbf{y}, \mathbf{y}, \mathbf{C})$ belongs to $L^{1}(\Omega \times[0, T])$ provided that $p \geq n$. 
Proof. The case $n=2$ is relatively easy and can be treated as, for instance, in [60, Page 918], so we limit ourself to the case $n \geq 3$.

The proof of ((i)) for the case $p \geq n$ is very similar to the proof of part ((ii)), so we will only treat the case $p<n$. Let $q=\frac{n p}{n-p}, \rho=\frac{p(n+2)}{n}$ and $\theta=\frac{2}{n+2} \in\left(0, \frac{1}{2}\right)$. By arguing as in [35, Chapter 2, page 213] we have

$$
L^{p}\left(0, T ; \mathbb{W}^{1, p}(\mathcal{Q})\right) \cap L^{\infty}\left(0, T ; L^{2}(\mathcal{Q})\right) \subset L^{p}\left(0, T ; L^{q}(\mathcal{Q})\right) \cap L^{\infty}\left(0, T ; L^{2}(\mathcal{Q})\right) \subset L^{\rho}([0, T] \times \mathcal{Q}),
$$

and

$$
\|\mathbf{v}\|_{L^{\rho}([0, T] \times \mathcal{Q})} \leq C\|\mathbf{v}\|_{L^{p}\left(0, T ; \mathbb{W}^{1, p}(\mathcal{Q})\right)}^{1-\theta}\|\mathbf{v}\|_{L^{\infty}\left(0, T ; L^{2}(\mathcal{Q})\right)}^{\theta},
$$

for any $\mathbf{v} \in L^{p}\left(0, T ; \mathbb{W}^{1, p}(\mathcal{Q})\right) \cap L^{\infty}\left(0, T ; L^{2}(\mathcal{Q})\right)$.

As above we can argue as in [35, Chapter 2, page 213] and derive that

$$
L^{2}\left(0, T ; \mathbb{H}^{1}(\mathcal{Q})\right) \cap L^{\infty}\left(0, T ; L^{2}(\mathcal{Q})\right) \subset L^{2}\left(0, T ; L^{\frac{2 n}{n-2}}(\mathcal{Q})\right) \cap L^{\infty}\left(0, T ; L^{2}(\mathcal{Q})\right) \subset L^{\alpha}([0, T] \times \mathcal{Q}),
$$

where $\alpha=\frac{2(n+2)}{n}$. Moreover, with the same $\theta$ as above

$$
\|\mathbf{v}\|_{L^{\alpha}([0, T] \times \mathcal{Q})} \leq C\|\mathbf{v}\|_{L^{2}\left(0, T ; \mathbb{H}^{1}(\mathcal{Q})\right)}^{1-\theta}\|\mathbf{v}\|_{L^{\infty}\left(0, T ; L^{2}(\mathcal{Q})\right)}^{\theta},
$$

for any $\mathbf{v} \in L^{p}\left(0, T ; \mathbb{W}^{1, p}(\mathcal{Q})\right) \cap L^{\infty}\left(0, T ; L^{2}(\mathcal{Q})\right)$.

Now since $b(\mathbf{u}, \mathbf{u}, \mathbf{v})=-b(\mathbf{u}, \mathbf{v}, \mathbf{u})$ for any $\mathbf{v} \in L^{p}\left(\Omega \times[0, T] ; \mathbb{V}_{1, p}\right)$ and $\frac{1}{p}+\frac{2}{\rho}$ for any $p \geq 1+\frac{2 n}{n+2}$, we derive by the Cauchy-Schwarz inequality that

$$
\begin{aligned}
\mathbb{E} \int_{0}^{T}|b(\mathbf{u}(t), \mathbf{u}(t), \mathbf{v}(t))| d t & \leq C \mathbb{E}\left[\|\mathbf{u}\|_{L^{\rho}([0, T] \times \mathcal{Q})}^{2}\|\nabla \mathbf{v}\|_{L^{p}([0, T] \times \mathcal{Q})}\right] \\
& \leq C \mathbb{E}\left[\|\mathbf{u}\|_{L^{p}\left(0, T ; \mathbb{W}^{1, p}(\mathcal{Q})\right)}^{2(1-\theta)}\|\mathbf{u}\|_{L^{\infty}\left(0, T ; L^{2}(\mathcal{Q})\right)}^{2 \theta}\|\mathbf{v}\|_{L^{p}\left(0, T ; \mathbb{V}_{1, p}\right)}\right] .
\end{aligned}
$$

Since $(\mathbf{u} ; \mathbf{B}) \in L^{r}(\Omega, C(0, T ; \mathbb{H})) \cap L^{r / 2}\left(\Omega, L^{p, 2}(0, T ; \mathbb{V})\right)$ for any $r \geq 2$ one can choose $r_{1}$ such that $\frac{2(1-\theta)}{r_{1}}+\frac{2 \theta}{r_{1}}+\frac{1}{p}=1$ and

$$
\begin{aligned}
\mathbb{E} \int_{0}^{T}|b(\mathbf{u}(t), \mathbf{u}(t), \mathbf{v}(t))| d t \leq C\left[\mathbb{E}\|\mathbf{u}\|_{L^{p}\left(0, T ; \mathbb{W}^{1, p}(\mathcal{Q})\right)}^{r_{1}}\right]^{\frac{2(1-\theta)}{r_{1}}} & {\left[\mathbb{E}\|\mathbf{u}\|_{L^{\infty}\left(0, T ; L^{2}(\mathcal{Q})\right)}^{2 r_{1}}\right]^{\frac{\theta}{r_{1}}} } \\
& \times\left[\mathbb{E}\|\mathbf{v}\|_{L^{p}\left(0, T ; \mathbb{V}_{1, p}\right)}^{p}\right]^{\frac{1}{p}} .
\end{aligned}
$$

With exactly the same argument we can prove the following estimate

$$
\begin{aligned}
\mathbb{E} \int_{0}^{T}|b(\mathbf{B}(t), \mathbf{B}(t), \mathbf{v}(t))| d t \leq C\left[\mathbb{E}\|\mathbf{B}\|_{L^{2}\left(0, T ; \mathbb{H}^{1}(\mathcal{Q})\right)}^{r_{1}}\right]^{\frac{2(1-\theta)}{r_{1}}} & {\left[\mathbb{E}\|\mathbf{B}\|_{L^{\infty}\left(0, T ; L^{2}(\mathcal{Q})\right)}^{2 r_{1}}\right]^{\frac{\theta}{r_{1}}} } \\
& \times\left[\mathbb{E}\|\mathbf{v}\|_{L^{p}\left(0, T ; \mathbb{V}_{1, p}\right)}^{p}\right]^{\frac{1}{p}},
\end{aligned}
$$

which is valid as long as $\frac{1}{p}+\frac{2}{\alpha} \leq 1$, i.e. $p \geq \frac{n+2}{4}$. We conclude easily the proof of part ((i)) with these last two estimates.

Proof of part ((ii)). Let

$$
\begin{gathered}
\gamma=\frac{p n^{2}}{[(p-2) n+2 p](n+2)}, \\
\frac{1}{q}=\gamma\left(\frac{1}{p}-\frac{1}{n}\right)+(1-\gamma) \frac{1}{2}
\end{gathered}
$$


Note that $n>\frac{n(n+2)}{2(n+1)}, \gamma \in(0,1)$ and $\frac{1}{q}+\frac{1}{2}+\frac{1}{\alpha} \leq 1$ as long as $p>\frac{n(n+2)}{2(n+1)}$. Hence, by the Cauchy-Schwarz and Gagliardo-Nirenberg inequalities we deduce that

$$
\mathbb{E} \int_{0}^{T}\left|\mathcal{B}_{2}(\mathbf{y}(t), \mathbf{y}(t), \mathbf{C}(t))\right| d t \leq C \mathbb{E}\left[\int_{0}^{T}|\mathbf{u}(t)|^{1-\gamma}\|\nabla \mathbf{u}(t)\|_{L^{p}(\mathcal{Q})}^{\gamma}\|\mathbf{B}(t)\|_{L^{\alpha}(\mathcal{Q})}|\nabla \mathbf{C}(t)| d t\right] .
$$

We deduce from this inequality that

$$
\begin{aligned}
\mathbb{E} \int_{0}^{T}\left|\mathcal{B}_{2}(\mathbf{y}(t), \mathbf{y}(t), \mathbf{C}(t))\right| d t \leq C \mathbb{E}[ & \|\mathbf{u}\|_{L^{\infty}\left(0, T ; L^{2}(\mathcal{Q})\right)}^{1-\gamma}\|\nabla \mathbf{u}\|_{L^{p}([0, T] \times \mathcal{Q})}^{\gamma} \\
& \left.\times\|\mathbf{B}\|_{L^{\alpha}([0, T] \times \mathcal{Q})}\|\nabla \mathbf{C}\|_{L^{2}([0, T] \times \mathcal{Q})}\right],
\end{aligned}
$$

provided that $\frac{\gamma}{p}+\frac{1}{2}+\frac{1}{\alpha} \leq 1$ which is true as long as $\gamma \leq \frac{n}{n+2}$, i.e. $p \geq n$. As before we can choose $r_{1}$ and $r_{2}$ such that $\frac{1-\gamma}{r_{1}}+\frac{\gamma}{2 r_{1}}+\frac{1-\theta}{r_{2}}+\frac{\theta}{2 r_{2}}+\frac{1}{2} \leq 1$ and

$$
\begin{aligned}
\mathbb{E} \int_{0}^{T}\left|\mathcal{B}_{2}(\mathbf{y}(t), \mathbf{y}(t), \mathbf{C}(t))\right| d t \leq \mathbb{E}\left[\|\mathbf{B}\|_{L^{2}\left(0, T ; \mathbb{H}^{1}(\mathcal{Q})\right)}^{r_{2}}\right]^{\frac{1-\theta}{r_{2}}}\left[\mathbb{E}\|\mathbf{B}\|_{L^{\infty}\left(0, T ; L^{2}(\mathcal{Q})\right)}^{2 r_{2}}\right)^{\frac{\theta}{2 r_{2}}} \\
\times \mathbb{E}\left[\|\mathbf{u}\|_{L^{p}\left(0, T ; \mathbb{W}^{1, p}(\mathcal{Q})\right)}^{r}\right]^{\frac{1-\gamma}{r}}\left[\mathbb{E}\|\mathbf{u}\|_{L^{\infty}\left(0, T ; L^{2}(\mathcal{Q})\right)}^{2 r_{1}}\right)^{\frac{\gamma}{2 r_{1}}} \\
\times\left[\mathbb{E}\|\mathbf{C}\|_{L^{2}\left(0, T ; \mathbb{V}_{2}\right)}^{2}\right]^{\frac{1}{2}}
\end{aligned}
$$

provided $p \geq n$. This last estimate ends the proof of our lemma.

Proof of Proposition 3.18. We will use the method of monotonicity (see, for instance, [47, Chapitre 3, Section 3, p 103 ] to prove Proposition 3.18.

Let $\left\{\Psi^{j} ; j=1,2, \ldots\right\}$ be the orthogonal basis of $\mathbb{H}$ defined on page 12 . From Lemma 3.5 we deduce that for any $j=1, \ldots, m_{k}$

$$
\begin{aligned}
\left(\overline{\mathbf{y}}^{m_{k}}(T), \Psi^{j}\right) & =\left(\mathbf{y}_{0}^{m_{k}}, \Psi^{j}\right)-\int_{0}^{T}\left\langle P_{m_{k}} \mathcal{A} \overline{\mathbf{y}}^{m_{k}}(s)+P_{m_{k}} \mathcal{B}\left(\overline{\mathbf{y}}^{m_{k}}(s), \overline{\mathbf{y}}^{m_{k}}(s)\right), \Psi^{j}\right\rangle d s \\
& +\int_{0}^{T}\left\langle P_{m_{k}} f\left(\overline{\mathbf{y}}^{m_{k}}(s), s\right), \Psi^{j}\right\rangle d s+\int_{0}^{T}\left\langle P_{m_{k}} g\left(\overline{\mathbf{y}}^{m_{k}}(s), s\right), \Psi^{j}\right\rangle d W_{m_{k}} .
\end{aligned}
$$

Thanks to (93) it follows that the righthand side the above equation converges weakly to $\left\langle\mathcal{M}(T), \Psi^{j}\right\rangle$ in $L^{2}(\Omega, \mathbb{R})$ which is equal to $\left(\mathbf{y}(T), \Psi^{j}\right)$ for any $j \geq 1$. Hence, we have just established that

$$
\beta=\mathbf{y}(T) \text { in } L^{2}(\Omega, \mathbb{H}) .
$$

Now, it follows from Eq. (12) of Lemma 2.3 that for any $\mathbf{v} \in L^{2}\left(\Omega, L^{p, 2}(0, T, \mathbb{V})\right)$

$$
2 \mathbb{E} \int_{0}^{T}\left\langle\mathcal{A} \overline{\mathbf{y}}^{m_{k}}(s), \overline{\mathbf{y}}^{m_{k}}(s)\right\rangle d s \geq 2 \mathbb{E} \int_{0}^{T}\left\langle\mathcal{A} \overline{\mathbf{y}}^{m_{k}}(s), \mathbf{v}(s)\right\rangle d s+2 \mathbb{E} \int_{0}^{T}\left\langle\mathcal{A} \mathbf{v}(s), \overline{\mathbf{y}}^{m_{k}}(s)-\mathbf{v}(s)\right\rangle d s .
$$

By assumption $p \geq \max \left(1+\frac{2 n}{n+2}, \frac{n+2}{4}, n\right)$, hence thanks to Lemma 3.19 we can apply Itô's formula given in [48, Theorem I.3.3.2, page 147] and we derive that

$$
\begin{array}{r}
2 \mathbb{E} \int_{0}^{T}\left\langle\mathcal{A} \overline{\mathbf{y}}^{m_{k}}(s), \overline{\mathbf{y}}^{m_{k}}(s)\right\rangle d s=\left|\mathbf{y}_{0}^{m_{k}}\right|^{2}-\mathbb{E}\left|\overline{\mathbf{y}}^{m_{k}}(T)\right|^{2}+2 \mathbb{E} \int_{0}^{T}\left\langle P_{m_{k}} f\left(\overline{\mathbf{y}}^{m_{k}}(s), s\right), \overline{\mathbf{y}}^{m_{k}}(s)\right\rangle d s \\
+\mathbb{E} \int_{0}^{T}\left\|P_{m_{k}} g\left(\overline{\mathbf{y}}^{m_{k}}(s), s\right)\right\|_{J_{2}}^{2} d s,
\end{array}
$$


and

$$
2 \mathbb{E} \int_{0}^{T}\langle\Gamma(s), \mathbf{y}(s)\rangle d s=\left|\mathbf{y}_{0}\right|^{2}-\mathbb{E}|\mathbf{y}(T)|^{2}+2 \mathbb{E} \int_{0}^{t}\langle f(\mathbf{y}(s), s), \mathbf{y}(s)\rangle d s+\mathbb{E} \int_{0}^{t}\|g(\mathbf{y}(s), s)\|_{J_{2}}^{2} d s .
$$

From these last three estimates we infer that

$$
\begin{array}{r}
2 \mathbb{E} \int_{0}^{T}\left\langle\mathcal{A} \overline{\mathbf{y}}^{m_{k}}(s), \mathbf{v}(s)\right\rangle d s-2 \mathbb{E} \int_{0}^{T}\langle\Gamma(s), \mathbf{y}(s)\rangle d s+2 \mathbb{E} \int_{0}^{T}\left\langle\mathcal{A v}(s), \overline{\mathbf{y}}^{m_{k}}(s)-\mathbf{v}(s)\right\rangle d s \leq \sum_{i=1}^{4} J^{i, k} \\
+\mathbb{E}\left(|\mathbf{y}(T)|^{2}-\left|\overline{\mathbf{y}}^{m_{k}}(T)\right|^{2}\right),
\end{array}
$$

where

$$
\begin{aligned}
J^{1, k} & :=\left|\overline{\mathbf{y}}_{0}^{m_{k}}\right|^{2}-\left|\mathbf{y}_{0}\right|^{2} \\
J^{2, k} & :=2 \mathbb{E} \int_{0}^{T}\left\langle f(\mathbf{y}(s), s), \mathbf{y}(s)-\overline{\mathbf{y}}^{m_{k}}(s)\right\rangle d s \\
J^{3, k} & :=2 \mathbb{E} \int_{0}^{T}\left\langle f(\mathbf{y}(s), s)-P_{m_{k}} f\left(\overline{\mathbf{y}}^{m_{k}}(s), s\right), \overline{\mathbf{y}}^{m_{k}}(s)\right\rangle d s \\
J^{4, k} & :=\mathbb{E} \int_{0}^{T}\left\|P_{m_{k}} g\left(\overline{\mathbf{y}}^{m_{k}}(s), s\right)\right\|_{J_{2}}^{2} d s-\mathbb{E} \int_{0}^{T} \| g\left(\mathbf{y}(s), s \|_{J_{2}}^{2} d s .\right.
\end{aligned}
$$

It follows from (43), (86), (91) and (96) that $\lim _{k \rightarrow \infty} \sum_{i=1}^{4} J^{i, k}=0$, and since $\overline{\mathbf{y}}^{m_{k}}(T) \rightarrow \mathbf{y}(T)$ weak in $L^{2}(\Omega, \mathbb{H})$ we also infer that

$$
\liminf _{k \rightarrow \infty} \mathbb{E}\left(|\mathbf{y}(T)|^{2}-\left|\overline{\mathbf{y}}^{m_{k}}(T)\right|^{2}\right) \leq 0 .
$$

We easily infer from these last two remarks and by passing to the limit in (106) that

$$
2 \mathbb{E} \int_{0}^{T}\langle\Gamma(s)-\mathcal{A} \mathbf{v}(s), \mathbf{v}(s)-\mathbf{y}(s)\rangle d s \leq 0
$$

for any $\mathbf{v} \in L^{2}\left(\Omega, L^{p, 2}(0, T, \mathbb{V})\right)$. Let $\Psi \in L^{2}\left(\Omega, L^{p, 2}(0, T, \mathbb{V})\right)$ and $\theta>0$. By taking $\mathbf{v}=\mathbf{y} \pm \theta \Psi$, we derive from the last estimate that

$$
2 \mathbb{E} \int_{0}^{T}\langle\Gamma(s)-\mathcal{A}(\mathbf{y}(s) \pm \theta \Psi(s)), \pm \Psi(s)\rangle d s \leq 0,
$$

from which along the hemicontinuity of $\mathcal{A}$ we conclude the proof of the proposition.

Proposition 3.20. Let $\mathcal{N}$ be set the null sets of $\mathcal{F}$ - Let $\mathbb{F}=\left\{\mathcal{F}_{s}: s \in[0, T]\right\}$, where the $\sigma$-algebra $\mathcal{F}_{s}$ is defined by

$$
\mathcal{F}_{s}:=\sigma(\sigma(\mathbf{y}(r), W(r) ; r \in[0, s]) \cup \mathcal{N}) .
$$

The stochastic process $W$ is a $\mathbb{F}$-cylindrical Wiener process on $\mathbb{H}$.

Proof. This follows from Proposition 3.11 and Remark 2.8.

Now we will end the proof of our first main result.

Proof of Theorem 3.3. Now from Proposition 3.13, Proposition 3.17, Proposition 3.18 and Proposition 3.20 we infer that the system $\{(\Omega, \mathcal{F}, \mathbb{F}, \mathbb{P}),(\mathbf{y}, W)\}$ is a martingale solution to (38)-(39) in the sense of Definition 3.1. This ends the proof of the existence theorem. 


\section{Exponential DeCAy of the WEAK SOlution}

In the present section, we are interested in the asymptotic behavior of weak probabilistic solutions of (38)-(39) so we will assume the existence of such solution.

First, note that

$$
\begin{aligned}
\langle\mathcal{A} \mathbf{y}, \mathbf{y}\rangle & =\left\langle\mathcal{A}_{p} \mathbf{u}, \mathbf{u}\right\rangle+\left\langle\mathcal{A}_{2} \mathbf{B}, \mathbf{B}\right\rangle \\
& \geq \tilde{\nu}_{3}\left(\|\mathbf{u}\|_{1}^{2}+\|u\|_{1, p}^{p}\right)+\|\mathbf{B}\|_{2}^{2} \\
& \geq \tilde{\nu}_{3} \mathcal{P}|\mathbf{u}|^{2}+\mathcal{P}|\mathbf{B}|^{2}
\end{aligned}
$$

where $\mathcal{P}$ is the constant in Poincaré's inequality. Setting $\tilde{\mu}=\min \left(\tilde{\nu}_{3} \mathcal{P}, \mathcal{P}\right)$, then

$$
\langle\mathcal{A y}, \mathbf{y}\rangle \geq \tilde{\mu}|\mathbf{y}|^{2} .
$$

Throughout this section we suppose that there exist positive constants $\theta, M_{\alpha}, M_{\beta}, C_{f}$, and two integrable functions $\alpha(.) \beta($.$) satisfying$

$$
0<\alpha(t) \leq M_{\alpha} e^{-\theta t}, \quad 0<\beta(t) \leq M_{\beta} e^{-\theta t}
$$

and

$$
\langle f(\mathbf{y}, t), \mathbf{y}\rangle \leq \alpha(t)+\left(C_{f}+\beta(t)\right)|\mathbf{y}|^{2},
$$

for any $t \in[0, \infty)$ and $\mathbf{y} \in \mathbb{H}$.

We also assume that there exist positive constants $\zeta, M_{\delta}, M_{\gamma}$ and two positive functions $\gamma(),. \delta($.$) such that$

and

$$
\delta(t) \leq M_{\delta} e^{-\theta t}, \quad \gamma(t) \leq M_{\gamma} e^{-\theta t}
$$

$$
\|g(\mathbf{y}, t)\|_{J_{2}}^{2} \leq \gamma(t)+(\zeta+\delta(t))|\mathbf{y}|^{2}
$$

for any $t \in[0, \infty)$ and $\mathbf{y} \in \mathbb{H}$.

We also suppose that

$$
2 \tilde{\mu}>2 C_{f}+\zeta .
$$

Theorem 4.1. Under the conditions (108)-(112) any weak solution to (38)-(39) converges to zero almost surely exponentially.

Proof. Recall that

$$
\mathbf{y}(t)=\mathbf{y}_{0}-\int_{0}^{t}[\mathcal{A} \mathbf{y}(s)+\mathcal{B}(\mathbf{y}(s), \mathbf{y}(s))] d s+\int_{0}^{t} f(\mathbf{y}(s), s) d s+\int_{0}^{t} g(\mathbf{y}(s), s) d W,
$$

from which and Itô's formula (see, for instance, [48, Theorem I.3.3.2, page 147] )we derive that

$$
\begin{array}{r}
|\mathbf{y}(t)|^{2}=\left|\mathbf{y}_{0}\right|^{2}-2 \int_{0}^{t}\langle\mathcal{A} \mathbf{y}(s)-f(\mathbf{y}(s), s), \mathbf{y}(s)\rangle d s+\int_{0}^{t}\|g(\mathbf{y}(s), s)\|_{J_{2}}^{2} d s \\
+2 \int_{0}^{t}(g(\mathbf{y}(s), s), \mathbf{y}(s)) d W .
\end{array}
$$

Since $2 \tilde{\mu}>2 C_{f}+\zeta$ we can choose a constant $a \in(0, \theta)$ such that $2 \tilde{\mu}>2 C_{f}+\zeta+a$. Hence, Itô's formula implies

$$
\begin{array}{r}
e^{a t}|\mathbf{y}(t)|^{2}=\left|\mathbf{y}_{0}\right|^{2}-2 \int_{0}^{t}\left\langle e^{a s} \mathcal{A} \mathbf{y}(s)\right. \\
-f(\mathbf{y}(s), s), \mathbf{y}(s)\rangle d s+\left.\int_{0}^{t} e^{a s}|| g(\mathbf{y}(s), s)\right|_{J_{2}} ^{2} d s \\
+a \int_{0}^{t} e^{a s}|\mathbf{y}(s)|^{2} d s+2 \int_{0}^{t}(g(\mathbf{y}(s), s), \mathbf{y}(s)) d W .
\end{array}
$$


Since the mathematical expectation of the last term of the right hand side of this equation vanishes, then

$$
\begin{array}{r}
e^{a t} \mathbb{E}|\mathbf{y}(t)|^{2}=\left|\mathbf{y}_{0}\right|^{2}-2 \int_{0}^{t} \mathbb{E}\left\langle e^{a s} \mathcal{A y}(s)-f(\mathbf{y}(s), s), \mathbf{y}(s)\right\rangle d s+\int_{0}^{t} e^{a s} \mathbb{E}|| g(\mathbf{y}(s), s) \|_{J_{2}}^{2} d s \\
+a \int_{0}^{t} e^{a s} \mathbb{E}|\mathbf{y}(s)|^{2} d s
\end{array}
$$

Therefore, we can derive from (113) and (107) that

$$
\begin{aligned}
e^{a t} \mathbb{E}|\mathbf{y}(t)|^{2} \leq \int_{0}^{t}\left(2 M_{\beta}+2 M_{\delta}\right) e^{(a-\theta) s}|\mathbf{y}(s)|^{2} d s & +\left(2 C_{f}+\zeta+a-2 \tilde{\mu}\right) \int_{0}^{t} e^{a s}|\mathbf{y}(s)|^{2} d s \\
& +\left|\mathbf{y}_{0}\right|^{2}+\int_{0}^{t}\left(2 M_{\alpha}+2 M_{\gamma}\right) e^{(a-\theta) s} d s .
\end{aligned}
$$

By invoking Gronwall's lemma we can infer the existence of $M_{0}=M_{0}\left(\left|\mathbf{y}_{0}\right|^{2}\right)$ such that

$$
\mathbb{E}|\mathbf{y}(t)|^{2} \leq M_{0} e^{-a t},
$$

for any $t \geq 0$.

Now let $N$ be a positive integer. Itô's formula yields

$$
\begin{array}{r}
|\mathbf{y}(t)|^{2}=|\mathbf{y}(N)|^{2}-2 \int_{N}^{t}\langle\mathcal{A y}(s)-f(\mathbf{y}(s), s), \mathbf{y}(s)\rangle d s+\int_{N}^{t}\|g(\mathbf{y}(s), s)\|_{J_{2}}^{2} d s \\
+2 \int_{N}^{t}(g(\mathbf{y}(s), s), \mathbf{y}(s)) d W .
\end{array}
$$

Owing to Burkhölder-Davis-Gundy's, Cauchy-Schwarz's and Cauchy's inequalities we have

$$
\begin{aligned}
\mathbb{E} \sup _{N \leq t \leq N+1}\left|\int_{N}^{t}(g(\mathbf{y}(s), s), \mathbf{y}(s)) d W\right| & \leq \eta_{1} \mathbb{E}\left(\int_{N}^{N+1}|\mathbf{y}(s)|^{2}\|g(\mathbf{y}(s), s)\|_{J_{2}}^{2} d s\right)^{\frac{1}{2}}, \\
& \leq \eta_{1} \mathbb{E}\left(\sup _{N \leq t \leq N+1}|\mathbf{y}(t)|^{2} \int_{N}^{N+1} \| g\left(\mathbf{y}(s), s \|_{J_{2}}^{2} d s\right)^{\frac{1}{2}},\right. \\
& \leq \eta_{2} \int_{N}^{N+1} \mathbb{E}\|g(\mathbf{y}(s), s)\|_{J_{2}}^{2} d s+\frac{1}{2} \mathbb{E} \sup _{N \leq t \leq N+1}|\mathbf{y}(t)|^{2},
\end{aligned}
$$

where $\eta_{1}, \eta_{2}>0$. From this we deduce that

$$
\begin{array}{r}
\mathbb{E} \sup _{N \leq t \leq N+1}|\mathbf{y}(t)|^{2} \leq 2 \int_{N}^{N+1}\left[\alpha(s)+\left(C_{f}+\beta(s)\right) \mathbb{E}|\mathbf{y}(s)|^{2}\right] d s \\
+ \\
+\mathbb{E}|\mathbf{y}(N)|^{2}-2 \tilde{\mu} \int_{N}^{N+1} \mathbb{E}|\mathbf{y}(s)|^{2} d s \\
+\left(1+\eta_{2}\right) \int_{N}^{N+1} \mathbb{E}|| g(\mathbf{y}(s), s) \|_{J_{2}}^{2} d s .
\end{array}
$$

The assumptions on $f$ and $g$ imply

$$
\begin{array}{r}
\mathbb{E} \sup _{N \leq t \leq N+1}|\mathbf{y}(t)|^{2} \leq \mathbb{E}|\mathbf{y}(N)|^{2}+\left(-2 \tilde{\mu}+2 C_{f}+\zeta\right) \int_{N}^{N+1} \mathbb{E}|\mathbf{y}(s)|^{2} d s \\
\quad+\int_{N}^{N+1}\left[2 \alpha(s)+\left(1+\eta_{2}\right) \gamma(s)\right] d s \\
+\int_{N}^{N+1}\left[2 \beta(s)+\eta_{2}(\zeta+\delta(s))\right] \mathbb{E}|\mathbf{y}(s)|^{2} d s
\end{array}
$$


Thanks to (115) there exists a positive constant $M_{1}=M_{1}\left(\left|\mathbf{y}_{0}\right|^{2}\right)$ such that

$$
\mathbb{E} \sup _{N \leq t \leq N+1}|\mathbf{y}(s)|^{2} \leq M_{1} e^{-a N} .
$$

Finally, the results follows from Borel-Cantelli's lemma.

Remark 4.2. The conditions (108)-(111) correspond to a modified MHD system driven by external forces that decay exponentially with time. This situation is very difficult to find in practical experiment, and hence it is not very realistic. A set of conditions which could be more realistic than (108)-(111) is the following.

Assume that there exist positive constants $M_{\alpha}, M_{\beta}, M_{\gamma}, M_{\delta}$ such that

$$
\langle f(\mathbf{y}), \mathbf{y}\rangle \leq M_{\alpha}+M_{\beta}|\mathbf{y}|^{2}
$$

and

$$
\|g(\mathbf{y}, t)\|_{J_{2}}^{2} \leq M_{\gamma}+M_{\delta}|\mathbf{y}|^{2},
$$

for any $\mathbf{y} \in \mathbb{H}$. Under these new conditions one can prove that Theorem 4.1 remains true provided that $\tilde{\mu}>M_{\alpha}+M_{\beta}+M_{\beta}+M_{\delta}$. A situation which correspond to these new assumptions is the case where we have a damping-like term (i.e., $f(\mathbf{y})=M_{\beta} \mathbf{y}$ ) and additive noise of the form $Q d W$ where $Q \in J_{2}(\mathbb{H})$.

\section{ACKNOWLEDGMENTS}

The authors gratefully acknowledge the careful reading of the manuscript by the reviewers, their comments and suggestions which have greatly improved the paper. The first named author is very grateful to the financial support from the Austrian Science Foundation through the Lise Meitner Program M1487. The research of the second named author is supported by the National Research Foundation of South Africa.

\section{REFERENCES}

[1] S. Albeverio, Z. Brzeźniak and J.-L. Wu. Existence of global solutions and invariant measures for stochastic differential equations driven by Poisson type noise with non-Lipschitz coefficients. J. Math. Anal. Appl. 371(1):309-322, 2010.

[2] V. Barbu, G. Da Prato. Existence and ergodicity for the two-dimensional stochastic magneto-hydrodynamics equations. Appl. Math. Optim. 56(2):145-168, 2007.

[3] H. Bellout, F. Bloom and J. Necas. Young measure-valued solutions for Non-Newtonian incompressible fluids. Communication in Partial Differential Equations. 19(11\& 12):1763-1803, 1994.

[4] Bensoussan A., Temam, R.: Équations stochastiques du type Navier-Stokes. J. Functional Analysis 13:95222, 1973.

[5] P. Billingsley. Convergence of probability measures. Second edition. Wiley Series in Probability and Statistics: Probability and Statistics. A Wiley-Interscience Publication. John Wiley \& Sons, Inc., New York, 1999.

[6] D. Biskamp. Magnetohydrodynamical Turbulence. Cambridge University Press, Cambridge, 2003.

[7] Z. Brzeźniak. On stochastic convolutions in Banach spaces and applications. Stoch. Stoch. Rep. 61:245-295, 1997.

[8] Z. Brzeźniak and L. Debbi. On stochastic Burgers equation driven by a fractional Laplacian and space-time white noise. Stochastic differential equations: Theory and applications, Interdiscip. Math. Sci., 2, World Sci. Publ., pages 135-167, 2007.

[9] Z. Brzeźniak, B. Goldys and T. Jegaraj. Weak solution of a Stochastic Landau-Lifshitz-Gilbert Equation. Appl. Math. Res. Express, pp 33, 2012.

[10] Z. Brzeźniak, B. Maslowski and J. Seidler. Stochastic nonlinear beam equation, Probab. Theory Relat. Fields. 132(2):119-144, 2005.

[11] Z. Brzezńiak and E. Motyl. Existence of a martingale solution of the stochastic Navier-Stokes equations in unbounded 2D and 3D domains. J. Differential Equations 254(4):1627-1685, 2013.

[12] Z. Brzeźniak, S. Peszat. Stochastic two dimensional Euler equations. Ann. Probab. 29(4): 1796-1832, 2001.

[13] T. Caraballo, J.A. Langa and T. Taniguchi. The exponential behaviour and stabilizability of stochastic 2DNavier-Stokes equations. J. Differential Equations. 179(2):714-737, 2002. 
[14] T. Caraballo, A.M. Márquez-Durán and J. Real. The asymptotic behaviour of a stochastic 3D LANS- $\alpha$ model. Appl. Math. Optim. 53(2):141-161, 2006.

[15] S. Chandrasekhar. Hydrodynamic and Hydromagnetic Stability. Dover, 1981.

[16] G. Da Prato and J. Zabczyk. Stochastic Equations in Infinite Dimensions. Cambridge University Press, 1992.

[17] G. Da Prato and A. Debussche 2D stochastic Navier-Stokes equations with a time-periodic forcing term. $J$. Dynam. Differential Equations 20(2):301-335, 2008.

[18] B. Desjardins and C. Le Bris. Remarks on a nonhomogeneous model of magnetohydrodynamics. Differential Integral Equations. 11(3):377-394, 1998.

[19] G. Deugoue and M. Sango. On the Stochastic 3D Navier-Stokes- $\alpha$ Model of Fluids Turbulence, Abstract and Applied Analysis, vol. 2009, Article ID 723236, 27 pages, 2009. doi:10.1155/2009/723236.

[20] G. Deugoue and M. Sango. On the Strong Solution for the 3D Stochastic Leray-Alpha Model, Boundary Value Problems, vol. 2010, Article ID 723018, 31 pages, 2010. doi:10.1155/2010/723018.

[21] G. Deugoue and M. Sango. Weak solutions to stochastic 3D Navier-Stokes- $\alpha$ model of turbulence: $\alpha$ Asymptotic behavior. Journal of Mathematical Analysis and Applications. 384:49-62, 2011.

[22] J.-F. Gerbeau, and C. Le Bris. Existence of solution for a density-dependent magnetohydrodynamic equation. Adv. Differential Equations 2(3):427-452, 1997.

[23] J.-F. Gerbeau, C. Le Bris, and T. Lelièvre. Mathematical methods for the Magnetohydrodynamics of Liquid Metals. Oxford University Press, New York, 2006.

[24] M. D. Gunzburger, O.A. Ladyzhenskaya, and J.S. Peterson. On the global unique solvability of initialboundary value problems for the coupled modified Navier-Stokes and Maxwell equations. J. Math. Fluid Mech. 6(4):462-482, 2004.

[25] M. D. Gunzburger and C. Trenchea. Analysis of an optimal control problem for the three-dimensional coupled modified Navier-Stokes and Maxwell equations. J. Math. Anal. Appl. 333(1):295-310, 2007.

[26] M. Hairer and J. C. Mattingly. A theory of hypoellipticity and unique ergodicity for semilinear stochastic PDEs. Electron. J. Probab. 16(23):658-738, 2011.

[27] N. Ikeda and S. Watanabe. Stochastic differential equations and diffusion processes. Second edition. Volume 24 of North-Holland Mathematical Library, North-Holland Publishing Co., Amsterdam/Kodansha, Ltd., Tokyo, 1989.

[28] T. Komorowski, S. Peszat and T. Szarek. On ergodicity of some Markov processes. Ann. Probab. 38(4):14011443,2010

[29] N. V. Krylov and B. L. Rozovskii. Stochastic Evolution Equations. Journal of Mathematical Sciences. 16(4): 1233-1277, 1981.

[30] O. Kallenberg. Foundations of modern probability. Probability and its Applications (New York). SpringerVerlag, New York, 1997.

[31] I. Karatzas and S. E. Shreve. Brownian motion and stochastic calculus. Second edition. Graduate Texts in Mathematics, 113. Springer-Verlag, New York, 1991.

[32] A. Kupiainen. Statistical theories of turbulence. In Advances in Mathematical Sciences and Applications. Gakkotosho, Tokyo, 2003.

[33] H. J. Kushner. Numerical Methods for Controlled Stochastic Delay Systems. Birkhäuser, Boston, 2008.

[34] O.A. Ladyzhenskaya and V. Solonnikov. Solution of some nonstationary magnetohydrodynamical problems for incompressible fluid. Trudy of Steklov Math. Inst. 69:115-173, 1960.

[35] J.-L. Lions. Quelques méthodes de résolution des problèmes aux limites non linéaires. Dunod; Gauthier-Villars, Paris, 1969.

[36] J. Málek, J. Necas, and M. Ruzicka. On weak solutions to a class of non-Newtonian incompressible fluids in bounded three-dimensional domains: the case $p \geq 2$. Adv. Differential Equations 6(3):257-302, 2001.

[37] J. Málek, J. Necas, M. Rokyta and M. Ruzicka. Weak and measure-valued solutions to evolutionary PDEs. Applied Mathematics and Mathematical Computation, 13. Chapman \& Hall, London, 1996.

[38] Málek, Josef; Nečas, Jindřich; Rajagopal, K. R. Global analysis of the flows of fluids with pressure-dependent viscosities. Arch. Ration. Mech. Anal. 165(3):243-269, 2002.

[39] Málek, J.; Rajagopal, K. R.; Růžička, M. Existence and regularity of solutions and the stability of the rest state for fluids with shear dependent viscosity. Math. Models Methods Appl. Sci. 5(6):789-812, 1995.

[40] Maslov, V. P.; Omelyanov, G. A. Three-scale expansion of the solution of equations of magnetohydrodynamics and Reynolds equations for a tokamak. Theoret. and Math. Phys. 98(2):202-211, 1994.

[41] Maslov, V. P. and Omelyanov, G. A. Rapidly oscillating asymptotic solution of equations of magnetohydrodynamics in the tokamak approximation. Theoret. and Math. Phys. 92(2): 879-895, 1993.

[42] M. Métivier. Stochastic Partial Differential Equations in Infinite Dimensional Spaces. Scuola Normale Superiore, Pisa, 1988. 
[43] R. Mikulevicius and B. L. Rozovskii. Stochastic Navier-Stokes Equations and Turbulent Flows. SIAM J. Math. Anal., 35(5):1250-1310, 2004.

[44] E. Motyl. Stochastic Navier-Stokes equations driven by Lévy noise in unbounded 3D domains. Potential Anal. 38(3): 863-912, 2013.

[45] E. Motyl. Stochastic hydrodynamic-type evolution equations driven by Lévy noise in 3D unbounded domains - abstract framework and applications.Stochastic Process. Appl. 124(6):2052-2097, 2014.

[46] A. L. Neidhardt. Stochastic Integrals in 2-uniformly smooth Banach Spaces. PhD Thesis, University of Wisconsin, 1978.

[47] E. Pardoux. Equations aux dérivées partielles stochastiques monotones. Thèse de Doctorat, Université ParisSud, 1975.

[48] E. Pardoux. Stochastic partial differential equations and filtering of diffusion processes. Stochastics 3(2):127167, 1979.

[49] K.R. Parthasarathy. Probability measures on metric spaces. Volume 3 of Probability and Mathematical Statistics, Academic Press, Inc., New York-London, 1967.

[50] S. Peszat and J. Zabczyk. Stochastic Partial Differential Equations with Levy Noise. An evolution equation approach. Encyclopedia of Mathematics and its Applications 113, Cambridge university Press, 2007.

[51] P. A. Razafimandimby and M. Sango. Weak Solutions of a Stochastic Model for Two-Dimensional Second Grade Fluids, Boundary Value Problems, vol. 2010, Article ID 636140, 47 pages, 2010. doi:10.1155/2010/636140.

[52] P. A. Razafimandimby. On Stochastic Models Describing the Motions of Randomly Forced Linear Viscoelastic Fluids, Journal of Inequalities and Applications, vol. 2010, Article ID 932053, 27 pages, 2010. doi:10.1155/2010/932053.

[53] P.A. Razafimandimby and M. Sango. Asymptotic behavior of solutions of stochastic evolution equations for second grade fluids. C. R. Math. Acad. Sci. Paris. 348(13-14):787-790, 2010.

[54] M. Riedle. Cylindrical Wiener processes. Séminaire de Probabilités XLIII, Lecture Notes in Math., 2006:191214, Springer, Berlin, 2011.

[55] Samokhin, V. N. On a system of equations in the magnetohydrodynamics of nonlinearly viscous media. Differential Equations 27(5):628-636, 1991.

[56] Samokhin, V. N. Existence of a solution of a modification of a system of equations of magnetohydrodynamics. Math. USSR-Sb. 72(2):373-385, 1992.

[57] V. N. Samokhin. Stationary problems of the magnetohydrodynamics of non-Newtonian media. Siberian Math. J. 33(4):654-662, 1993.

[58] V. N. Samokhin. The operator form and solvability of equations of the magnetohydrodynamics of nonlinearly viscous media. Differ. Equ. 36(6):904-910, 2000.

[59] M. Sango. Weak solutions for a doubly degenerate quasilinear parabolic equation with random forcing. Discrete Contin. Dyn. Syst. Ser. B, 7(4):885-905, 2007.

[60] M. Sango. Magnetohydrodynamic turbulent flows: Existence results. Physica D: Nonlinear Phenomena 239(12): 912-923, 2010.

[61] M. Sango. Density dependent stochastic Navier-Stokes equations with non Lipschitz random forcing. Reviews in Mathematical Physics 22(6):669-697, 2010.

[62] M. Sermange and R. Temam. Some mathematical questions related to the MHD equations. Comm. Pure Appl. Math. 36(5):635-664, 1983.

[63] J. Simon. Compact sets in the space $L^{p}(0 ; T ; B)$. Annali Mat. Pura Appl. 146(4):65-96, 1987.

[64] R. Situ. Theory of Stochastic Differential Equations with Jumps and Applications. Mathematical and Analytical Techniques with Applications to Engineering. Springer, New-York, 2005.

[65] A.V. Skorokhod. Studies in the theory of random processes. Translated from the Russian by Scripta Technica, Inc. Addison-Wesley Publishing Co., Inc., Reading, Mass., 1965.

[66] H. H. Sohrab. Basic real analysis. Birkhäuser Boston, Inc., Boston, 2003.

[67] S. S. Sritharan, P. Sundar. The stochastic magneto-hydrodynamic system. Infin. Dimens. Anal. Quantum Probab. Relat. Top. 2(2):241-265, 1999.

[68] W. A. Strauss, On continuity of functions with values in various Banach spaces. Pacific J. Math. 19: 543-551, 1966.

[69] L. Stupyalis. An initial-boundary value problem for a system of equations of magnetohydrodynamics. Lithuanian Math. J. 40(2):176-196, 2000.

[70] R. Temam. Navier-Stokes Equations. North-Holland, 1979.

[71] J. M. A. M. van Neerven Stochastic Evolution Equations. Lecture Lecture Notes of the Internet Seminar 2007/2009. Available on http://fa.its.tudelft.nl/isemwiki. (Access date 22 February 2013). 
[72] E. Zeidler. Nonlinear Functional Analysis and its Applications, II/A: Linear Monotone Operators. SpringerVerlag, New York, 1990.

(P. A. Razafimandimby) Department of Mathematics and Information Technology, Montanuniversität Leoben, Franz Josef Strasse 18, 8700 Leoben, Austria

(M. Sango) Department of Mathematics and Applied Mathematics, University of Pretoria, Lynwood Road, Pretoria 0002, South Africa

E-mail address, P.A. Razafimandimby: paul.razafimandimby@unileoben.ac.at

E-mail address, M. Sango: mamadou.sango@up.ac.za 